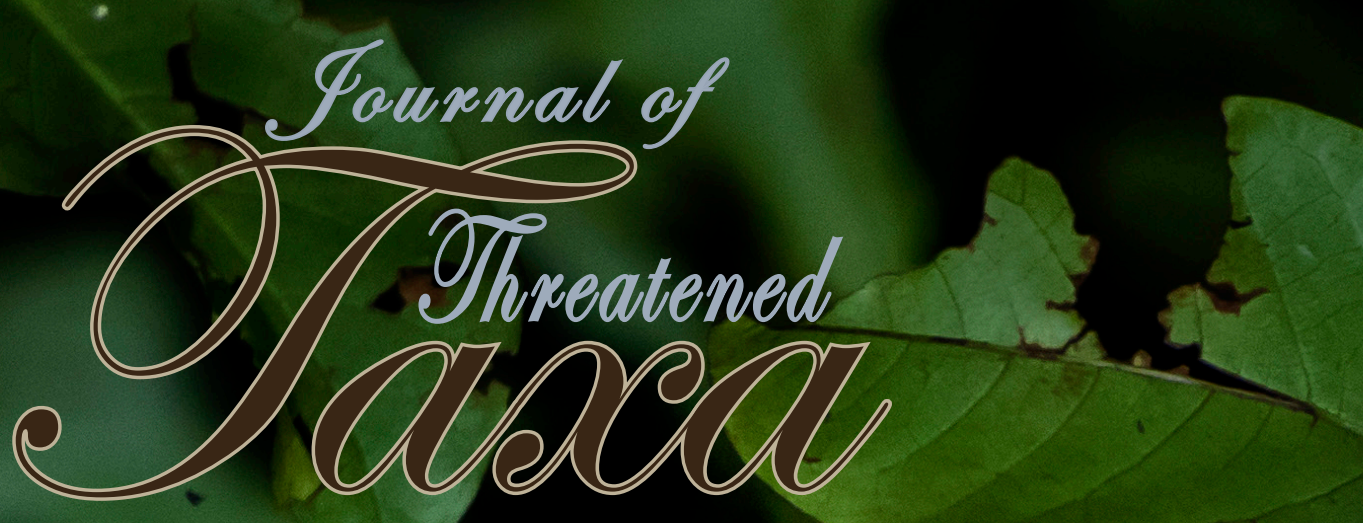

Building exidence for conservation glabally

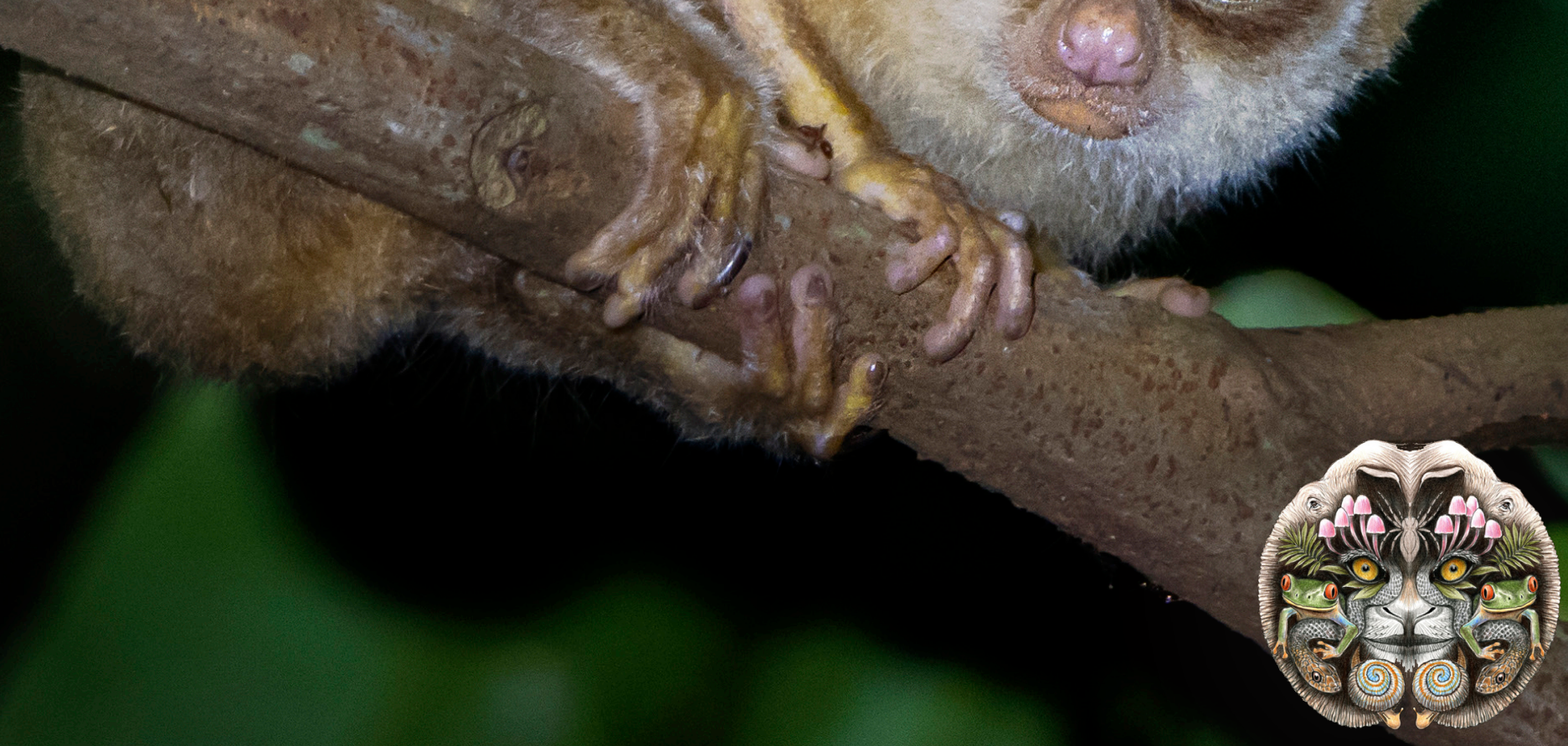

Open Access

$10.11609 /$ jott.2021.13.010.19431-19614 creven.threatenedtaxa.arg

26 September 2021 (Online \& Print) Val. 13 | Na. 11 | Pages: 19431-19674 


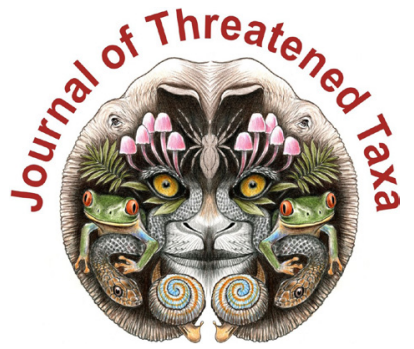

ISSN 0974-7907 (Online); ISSN $0974-7893$ (Print)

Publisher

Host

Wildlife Information Liaison Development Society

www.wild.zooreach.org

Zoo Outreach Organization www.zooreach.org

No. 12, Thiruvannamalai Nagar, Saravanampatti - Kalapatti Road, Saravanampatti, Coimbatore, Tamil Nadu 641035, India

Ph: +91 9385339863 | www.threatenedtaxa.org

Email: sanjay@threatenedtaxa.org

EDITORS

\section{Founder \& Chief Editor}

Dr. Sanjay Molur

Wildlife Information Liaison Development (WILD) Society \& Zoo Outreach Organization (ZOO)

12 Thiruvannamalai Nagar, Saravanampatti, Coimbatore, Tamil Nadu 641035, India

\section{Deputy Chief Edito}

Dr. Neelesh Dahanukar

Noida, Uttar Pradesh, India

\section{Managing Editor}

Mr. B. Ravichandran, WILD/ZOO, Coimbatore, India

\section{Associate Editors}

Dr. Mandar Paingankar, Government Science College Gadchiroli, Maharashtra 442605, India

Dr. Ulrike Streicher, Wildlife Veterinarian, Eugene, Oregon, USA

Ms. Priyanka Iyer, ZOO/WILD, Coimbatore, Tamil Nadu 641035, India

Dr. B. A. Daniel, $200 / \mathrm{WILD}$, Coimbatore, Tamil Nadu 641035, India

\section{Editorial Board}

Dr. Russel Mittermeier

Executive Vice Chair, Conservation International, Arlington, Virginia 22202, USA

\section{Prof. Mewa Singh Ph.D., FASc, FNA, FNASc, FNAPsy}

Ramanna Fellow and Life-Long Distinguished Professor, Biopsychology Laboratory, and Institute of Excellence, University of Mysore, Mysuru, Karnataka 570006, India; Honorary Professor, Jawaharlal Nehru Centre for Advanced Scientific Research, Bangalore; and Adjunct Professor, National Institute of Advanced Studies, Bangalore

\section{Stephen D. Nash}

Scientific Illustrator, Conservation International, Dept. of Anatomical Sciences, Health Sciences Center, T-8, Room 045, Stony Brook University, Stony Brook, NY 11794-8081, USA

\section{Dr. Fred Pluthero}

Toronto, Canada

\section{Dr. Priya Davidar}

Sigur Nature Trust, Chadapatti, Mavinhalla PO, Nilgiris, Tamil Nadu 643223, India

\section{Dr. Martin Fisher}

Senior Associate Professor, Battcock Centre for Experimental Astrophysics, Cavendish

Laboratory, JJ Thomson Avenue, Cambridge CB3 OHE, UK

\section{Dr. John Fellowe}

Honorary Assistant Professor, The Kadoorie Institute, 8/F, T.T. Tsui Building, The University of Hong Kong, Pokfulam Road, Hong Kong

\section{Prof. Dr. Mirco Solé}

Universidade Estadual de Santa Cruz, Departamento de Ciências Biológicas, Vice-coordenado do Programa de Pós-Graduação em Zoologia, Rodovia Ilhéus/Itabuna, Km 16 (45662-000)

Salobrinho, Ilhéus - Bahia - Brasil

\section{Dr. Rajeev Raghavan}

Professor of Taxonomy, Kerala University of Fisheries \& Ocean Studies, Kochi, Kerala, India

\section{English Editors}

Mrs. Mira Bhojwani, Pune, India

Dr. Fred Pluthero, Toronto, Canad

Mr. P. Ilangovan, Chennai, India

Web Maintenance

Mrs. Latha G. Ravikumar, ZOO/WILD, Coimbatore, India

\section{Typesetting}

Mr. Arul Jagadish, ZOO, Coimbatore, India

Mrs. Radhika, ZOO, Coimbatore, India

Mrs. Geetha, ZOO, Coimbatore India

\section{Fundraising/Communications}

Mrs. Payal B. Molur, Coimbatore, India

Subject Editors 2018-2020

Fungi

Dr. B. Shivaraju, Bengaluru, Karnataka, India

Dr. R.K. Verma, Tropical Forest Research Institute, Jabalpur, India

Dr. Vatsavaya S. Raju, Kakatiay University, Warangal, Andhra Pradesh, India

Dr. M. Krishnappa, Jnana Sahyadri, Kuvempu University, Shimoga, Karnataka, India

Dr. K.R. Sridhar, Mangalore University, Mangalagangotri, Mangalore, Karnataka, India

Dr. Gunjan Biswas, Vidyasagar University, Midnapore, West Bengal, India

Plants

Dr. G.P. Sinha, Botanical Survey of India, Allahabad, India

Dr. N.P. Balakrishnan, Ret. Joint Director, BSI, Coimbatore, India

Dr. Shonil Bhagwat, Open University and University of Oxford, UK

Prof. D.J. Bhat, Retd. Professor, Goa University, Goa, India

Dr. Ferdinando Boero, Università del Salento, Lecce, Italy

Dr. Dale R. Calder, Royal Ontaro Museum, Toronto, Ontario, Canada

Dr. Cleofas Cervancia, Univ. of Philippines Los Baños College Laguna, Philippines

Dr. F.B. Vincent Florens, University of Mauritius, Mauritius

Dr. Merlin Franco, Curtin University, Malaysia

Dr. V. Irudayaraj, St. Xavier's College, Palayamkottai, Tamil Nadu, India

Dr. B.S. Kholia, Botanical Survey of India, Gangtok, Sikkim, India

Dr. Pankaj Kumar, Kadoorie Farm and Botanic Garden Corporation, Hong Kong S.A.R., China

Dr. V. Sampath Kumar, Botanical Survey of India, Howrah, West Bengal, India

Dr. A.J. Solomon Raju, Andhra University, Visakhapatnam, India

Dr. Vijayasankar Raman, University of Mississippi, USA

Dr. B. Ravi Prasad Rao, Sri Krishnadevaraya University, Anantpur, India

Dr. K. Ravikumar, FRLHT, Bengaluru, Karnataka, India

Dr. Aparna Watve, Pune, Maharashtra, India

Dr. Qiang Liu, Xishuangbanna Tropical Botanical Garden, Yunnan, China

Dr. Noor Azhar Mohamed Shazili, Universiti Malaysia Terengganu, Kuala Terengganu, Malaysia Dr. M.K. Vasudeva Rao, Shiv Ranjani Housing Society, Pune, Maharashtra, India

Prof. A.J. Solomon Raju, Andhra University, Visakhapatnam, India

Dr. Mandar Datar, Agharkar Research Institute, Pune, Maharashtra, India

Dr. M.K. Janarthanam, Goa University, Goa, India

Dr. K. Karthigeyan, Botanical Survey of India, India

Dr. Errol Vela, University of Montpellier, Montpellier, France

Dr. P. Lakshminarasimhan, Botanical Survey of India, Howrah, India

Dr. Larry R. Noblick, Montgomery Botanical Center, Miami, USA

Dr. K. Haridasan, Pallavur, Palakkad District, Kerala, India

Dr. Analinda Manila-Fajard, University of the Philippines Los Banos, Laguna, Philippines

Dr. P.A. Sinu, Central University of Kerala, Kasaragod, Kerala, India

Dr. Afroz Alam, Banasthali Vidyapith (accredited A grade by NAAC), Rajasthan, India

Dr. K.P. Rajesh, Zamorin's Guruvayurappan College, GA College PO, Kozhikode, Kerala, India

Dr. David E. Boufford, Harvard University Herbaria, Cambridge, MA 02138-2020, USA

Dr. Ritesh Kumar Choudhary, Agharkar Research Institute, Pune, Maharashtra, India

Dr. Navendu Page, Wildlife Institute of India, Chandrabani, Dehradun, Uttarakhand, India

Invertebrates

Dr. R.K. Avasthi, Rohtak University, Haryana, India

Dr. D.B. Bastawade, Maharashtra, India

Dr. Partha Pratim Bhattacharjee, Tripura University, Suryamaninagar, India

Dr. Kailash Chandra, Zoological Survey of India, Jabalpur, Madhya Pradesh, India

Dr. Ansie Dippenaar-Schoeman, University of Pretoria, Queenswood, South Africa

Dr. Rory Dow, National Museum of natural History Naturalis, The Netherlands

Dr. Brian Fisher, California Academy of Sciences, USA

Dr. Richard Gallon, llandudno, North Wales, LL30 1UP

Dr. Hemant V. Ghate, Modern College, Pune, India

Dr. M. Monwar Hossain, Jahangirnagar University, Dhaka, Bangladesh

Mr. Jatishwor Singh Irungbam, Biology Centre CAS, Branišovská, Czech Republic

Dr. lan J. Kitching Natural History Museum, Cromwell Road, UK

Dr. George Mathew, Kerala Forest Research Institute, Peechi, India

Dr. John Noyes, Natural History Museum, London, UK

For Focus, Scope, Aims, and Policies, visit https://threatenedtaxa.org/index.php/JoTT/aims_scope
For Article Submission Guidelines, visit https://threatenedtaxa.org/index.php/JoTT/about/submissions
For Policies against Scientific Misconduct, visit https://threatenedtaxa.org/index.php/JoTT/policies_various 


\title{
Bivalves (Mollusca: Bivalvia) in Malaysian Borneo: status and threats
}

\author{
Abdulla-Al-Asif ${ }^{1}$ (D), Hadi Hamli ${ }^{2}$ (D), Abu Hena Mustafa Kamal ${ }^{3}$ (D), Mohd Hanafi Idris ${ }^{4}$ (D), \\ Geoffery James Gerusu ${ }^{5} \mathbb{D}$, Johan Ismail ${ }^{6} \mathbb{C}$ \& Muyassar H. Abualreesh ${ }^{7} \mathbb{D}$ \\ ${ }^{1.2,6}$ Department of Animal Science and Fishery, Faculty of Agricultural Science and Forestry, Universiti Putra Malaysia, \\ Bintulu Sarawak Campus, 97008 Bintulu, Sarawak, Malaysia. \\ ${ }^{3,4}$ Faculty of Fisheries and Food Science, Universiti Malaysia Terengganu, 21030 Kuala Nerus, Terengganu, Malaysia. \\ ${ }^{3}$ Centre for Environment and Sustainability, Presidency Education, 51 Panchlaish, Chittagong 4203, Bangladesh. \\ ${ }^{5}$ Department of Forestry Science, Faculty of Agricultural Science and Forestry, Universiti Putra Malaysia Bintulu Sarawak Campus, \\ Bintulu, 97008 Bintulu, Sarawak, Malaysia. \\ ${ }^{5,6}$ Institut Ekosains Borneo, Universiti Putra Malaysia Bintulu Sarawak Campus, Jalan Nyabau 97008 Bintulu, Sarawak, Malaysia Jalan \\ Nyabau 97008, Bintulu, Sarawak, Malaysia. \\ ${ }^{7}$ Department of Marine Biology, Faculty of Marine Sciences, King Abdualaziz University, P.O. Box 80207, Jeddah 21589, Saudi Arabia. \\ ${ }^{1}$ jessoreboyhemel@gmail.com, ${ }^{2}$ hadihamli@upm.edu.my (corresponding author), ${ }^{3}$ a.hena@umt.edu.my, ${ }^{4}$ hanafiidris@umt.edu.my, \\ ${ }^{5}$ geoffery@upm.edu.my, ${ }^{6}$ ijohan@upm.edu.my, ${ }^{7}$ mabulreesh1@kau.edu.sa
}

\begin{abstract}
Species checklists enlist the species existing within a distinct geographical biome and assist as an indispensable input for evolving conservation and administration strategies. The arenas of conservation ecology and biology face the challenge of exaggerated biodiversity, accredited to the non-recognition of taxonomic inconsistencies. The study's goals are to organize all scattered taxonomic information regarding bivalve molluscs from Malaysian Borneo, i.e. Sarawak and Sabah, under one umbrella. Available literature regarding Malaysian Borneo was reviewed. The published taxonomic data on bivalve species, conservation status, inconsistencies, habitats (marine, fresh, and brackish), research aspects, threats, and conservation strategies are presented. A critical review of the checklists and distributional records of the class Bivalvia from Malaysian Borneo and subsequent validation of species names with the World Register of Marine Species (WoRMS) database revealed that currently 76 bivalve species from 12 orders and other entities, 18 superfamilies, and 27 families have been recorded from the area. Twenty-six inconsistencies with WoRMS were found, and the corrected names are presented. The study indicates most of the enlisted bivalve species have not been evaluated by the IUCN Red List authority and have 'Least Concern' or 'Data Deficient' status for Malaysian Borneo. To date, published documents on conservation decision strategies and guidelines for future research are not good enough. Nevertheless, potential threats and their remedies for bivalves in the enriched Malaysian Borneo ecosystems are discussed herein.
\end{abstract}

Keywords. Biodiversity conservation, checklist, database, double-shelled molluscs, IUCN Red List, taxonomic inconsistency.

Citation: Abdulla-Al-Asif, H. Hamli, A.H.M. Kamal, M.H. Idris, G.J. Gerusu, J. Ismail \& M.H. Abualreesh (2021). Bivalves (Mollusca: Bivalvia) in Malaysian Borneo: status and threats. Journal of Threatened Taxa 13(11): 19553-19565. https://doi.org/10.11609/jott.7287.13.11.19553-19565

Copyright: (c) Abdulla-Al-Asif et al. 2021. Creative Commons Attribution 4.0 International License. JoTT allows unrestricted use, reproduction, and distribution of this article in any medium by providing adequate credit to the author(s) and the source of publication.

Funding: Ministry of Higher Education Malaysia.

Competing interests: The authors declare no competing interests.

Author details: ABDULLA-AL-ASIF currently working on seagrass and mangrove associated benthic fauna and their ecology. HADI HAMLI, GEOFFERY JAMES GERUSU and JOHAN ISMAIL works as senior lecturer in Universiti Putra Malaysia Bintulu Campus. ABU HeNA MUSTAFA KAMAL and MOHD HANAFI IDRIS works as associate professor in University of Malaysia Terengganu. MUYASSAR H. ABUALREESH works as assistant professor in Saudi Arabia.

Author contributions: AAA, and HH-Conceptualization, methodology, data collection, statistical analysis, and writing original-draft. AHMK, MHI, GJG, JI and MHA-writing, review and editing.

Acknowledgements: The research team acknowledges the Ministry of Higher Education Malaysia FRGS research grant code, FRGS/1/2018/WAB13/UPM/02/2. We would like to thank Md. Khurshid Alam Bhuiyan from Universidad de Cádiz for making the map of the study area. The research team thanks the Department of Animal Science and Fishery, University Putra Malaysia Bintulu Sarawak Campus, for technical support. Finally, we would like to express our gratitude for the efforts, suggestions, and insightful comments made by anonymous reviewers and editors on our manuscript, which significantly improved the current version of the paper.
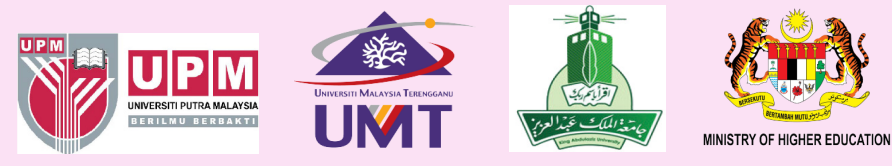


\section{INTRODUCTION}

The establishment of a database and checklist of regionally present species is crucial in managing and conserving them from alpha to global ecosystem (Amano \& Sutherland 2013). The lack of sufficient information at the local level regarding rare and/or endemic species potentially at risk of extinction may lead to strategies taken by different organizations, including the government, that are inadequate to avoid their extinction (Işik 2011). Nowadays, humankind faces some traumatic events, including the so-called "sixth extinction crisis". The previous five extinctions were caused by massive atmospheric, climatic, and universal phenomena, but the prediction of the next mass extinction is putting the finger on human interference in natural ecosystems (Braje \& Erlandson 2013).

At regional and local level, species decline faster than the prediction of ecologists (Collen et al. 2011; Işik 2011), but this can be modified to a sustainable level if the conservation efforts would focus on protecting certain species (Reydon 2019). For example, some aquatic animal recovered their extinction risk by the conservation approach with a proper policy, legislation, and effective conservation measures. Recently, the Bangladesh government and department of fisheries initiated the conservation of red fin mahseer (Tor tor Hamilton, 1822). The proper breeding program and management helped this species regain their confined population (Kabir et al. 2018). Similarly, the reproduction and conservation management of butter catfish Ompok pabda (Hamilton-Bouchanan, 1822) changes their IUCN status from 'Near Threatened' to 'Endangered' species (Chakraborty et al. 2010; IUCN Bangladesh 2015). Now, aquaculture is very extensive for Ompok pabda (Hamilton-Bouchanan, 1822) in Bangladesh and the Indian region (Chaklader et al. 2016; Alam et al. 2020).

Another is to protect specific areas with high biodiversity, including rare and/or endemic species. Now governments and third-party stakeholders recognize the value of biodiversity conservation, and they convey efforts, finances, and human resources to the conservation of nature. The first step in this process is to know the present status of biodiversity (Groves et al. 2002; Martin et al. 2016), where a checklist and relevant information are considered to be essential documents to step forward. Establishing a database of locally and regionally present species allows management of the national and transboundary continental conservation process (National Research Council 1992).

Bivalves (two valves), are abundant in marine, brackish and freshwater ecosystems, both infaunal and epifaunal in nature. Most are filter-feeders, but some are carnivores. They influence food webs and aquatic ecosystems via nutrient cycling and habitat modification and act as a bio-indicator (Vaughn \& Hoellein 2018). In many countries, bivalves are consumed by humans for which they are harvested from the wild, including freshwater and marine habitats (Köhler et al. 2012; Wijsman et al. 2019). As molluscs are rich in protein and fat, along with essential nutrients including vitamins and macro-micro nutrients, restaurants around the world serve them as delicious and luxury food (e.g., Venugopal \& Gopakumar 2017; Olivier et al. 2020). Bivalve shells, including the waste of such meals, are also used as buffer material for soil fixation; for instance, Korean scientists applied oyster waste to increase soil $\mathrm{pH}$ and other micromacro nutrients (Lee et al. 2008).

In East Malaysia (Sarawak and Sabah, including the federal territory of Labuan), bivalves are considered a delicacy, and highly nutritious consumable commodities (Hamli et al. 2012b). Some previous studies described the bivalve fauna of Peninsular Malaysia (Idris et al. 2012; Jasin 2015; Zieritz et al. 2016; Zieritz \& LopesLima 2018). Some studies have been conducted in the Malaysian province of Sarawak and Sabah covering different habitats, including mangroves (Hamli et al. 2015; Abu Hena et al. 2016), seagrass (Al-Asif et al. 2020), wetlands (Idris et al. 2021), and freshwater (Hamli et al. 2020). Noticeably, the bivalve species from freshwater environments have a more than four to six times higher risk of extinction than those in marine habitats (AgudoPadrón 2011).

Currently there is no monograph of bivalves (or molluscs in general) covering Malaysian Borneo. Thus far only a small fraction of the bivalve fauna of Malaysian Borneo has been recorded. The first bivalves from modern history of Malaysian Borneo were recorded from the Pantai river, Sarawak (Turner \& Santhakumaran 1989) and Sematan mangrove forest, Sarawak (Ashton et al. 2003), although the first record can be tracked back in 1791, from the Federal territory of Labuan (A small island near coast of Sarawak and Sabah in Malaysian Borneo), with the report of native bivalve species Marcia japonica (Gmelin, 1791) (reported as Venus japonica Gmelin, 1791) (Gmelin 1791). With time, the number of published documents (taxonomic and ecological studies) has increased, but the list of bivalve fauna from East Malaysia remains very incomplete. Numerous species are recorded in Huber (2010, 2015), but his records 'north Borneo' or just 'Borneo' are not specific enough to be included here. Similar taxonomic 
and conservation work was published on fish species of Bangladesh in which the implementation of conservation measures on local fish habitats was proposed (Parvez et al. 2019). Similarly, the current investigation intends making a checklist of bivalves in the Malaysian part of Borneo, including their conservation status. This study also discusses the existing research initiatives, future research prospective, and recommended measures toward conserving this vital living resource.

\section{MATERIALS AND METHODS}

The current study is based on published records regarding Malaysian Borneo (Sarawak and Sabah; Figure 1), including monographs, reviews, checklists, catalogues, posters, conference papers and posters, websites, and fishery reports from 1791 and 2020, but no additional material was collected. For each reported species the scientific names were confirmed based on the World Register of Marine Species (WoRMS) 2021 and MolluscaBase eds (2021) (validating unaccepted names, emendations, alternate, and representations).
The identifications were not checked for correctness. In most cases this was not possible, as most publications contain no photographs of the recorded species. New records should be accompanied by photographs as misidentifications are commonplace.

The species list comprises, orders, superfamilies, family name, accepted name, unaccepted names, and emendations. The contribution (\%) of different orders within the class Bivalvia and various superfamilies and families in the class was estimated. The statistical data, total species counts, and graphical presentation were analyzed using Microsoft Excel.

\section{RESULTS}

\section{Bivalve diversity}

A total of 76 species of bivalves from 12 orders/ infraclasses/ superorders/ subclasses, 18 superfamilies, and 27 families were reported from freshwater and marine habitats (seagrass meadow, intertidal, mangrove, freshwater, wetlands, and coastal region of Sarawak and Sabah) in Malaysian Borneo (Figure 2).

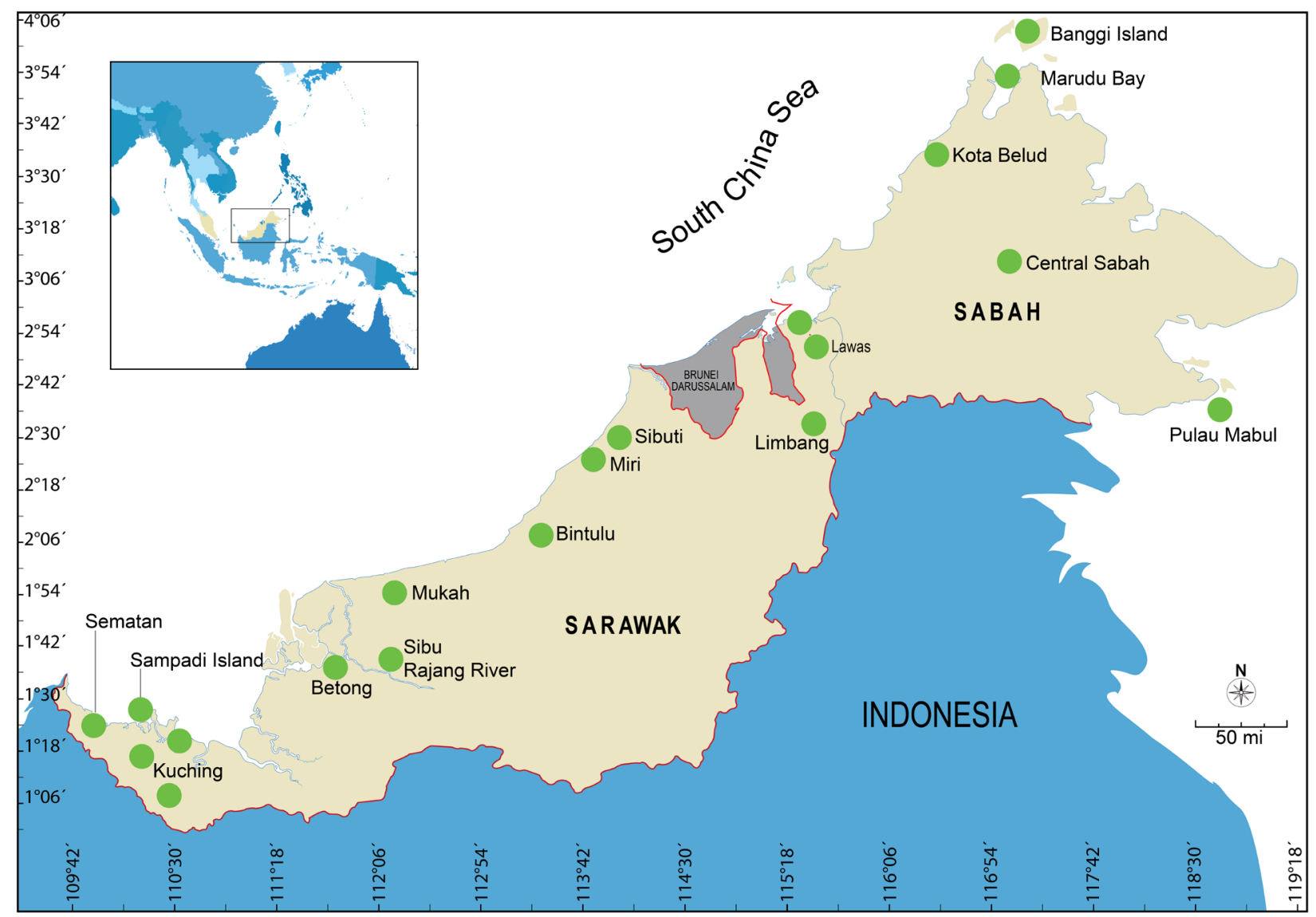

Figure 1. Map of the of the East Malaysian states showing Sarawak and Sabah (The green circles denote the areas covered the study). 


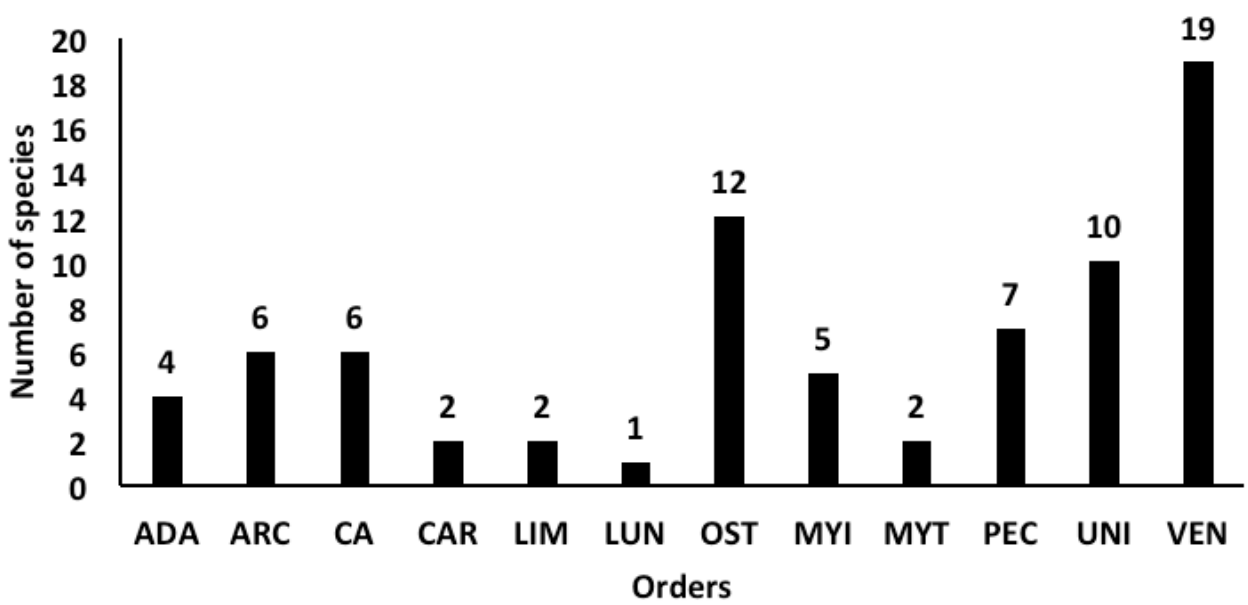

Figure 2. Number of Bivalves species based on the order recorded in Malaysian Borneo.

ADA-Adapedonta | ARC-Arcida | CA-Cardiida | CAR-Carditida | LIM-Limida | LUC-Lucinida | OST-Ostreida | MYI-Myida | MYTMytilida | PEC-Pectinida | UNI-Unionida | VEN-Venerida.

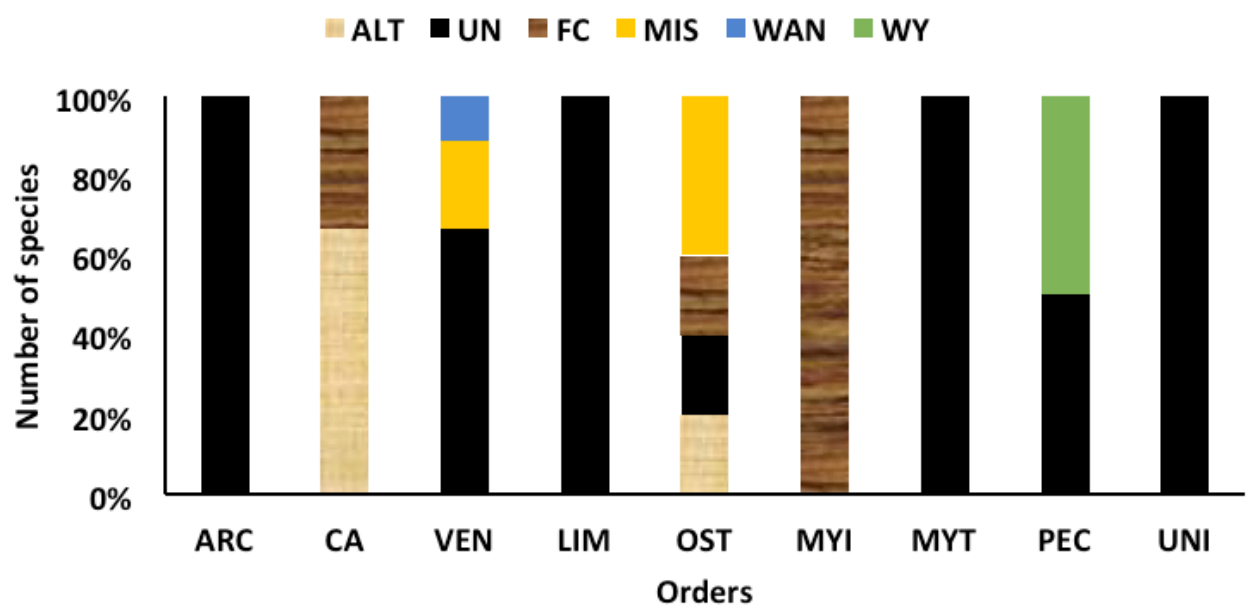

Figure 3. Extent of taxonomic inconsistencies in the orders of the class Bivalvia thus far recorded from Malaysian Borneo.

ArC-Arcida | Ca-Cardiida | Ven-Venerida | Lim_Limida | Ost-Ostreida | Myi-Myida | MYT_Mytilida | Pec-Pectinida | Uni-Unionida | ALT-Alternet representation | UN-Unaccepted | FC-Family changed | MIS-Misspelling | WAN-Wrong author name | WY-Wrong year.

A critical review of the published checklists revealed that the current literature included 26 incorrect names for bivalve species from nine orders/ infraclasses/ superorders/ subclasses and 14 families. Of these inconsistencies in the bivalve checklist over $53.84 \%$ (14 species) was due to names not accepted in WoRMS (2021), spelling mistakes (15.38\%; 4 entities), alternative representation and inconsistency in family name (both $11.54 \%$; 3 entities each), and inconsistencies in author and year (both 3.85 \%; 1 entity each) (Figure 3).

\section{Knowledge gap on bivalve research in Malaysian Borneo}

In the current century, macro benthic surveys were first conducted in Malaysian territory in 1981
(Morris \& Purchon 1981; Way \& Purchon 1981). In East Malaysia, Turner \& Santhakumaran (1989) and Ashton et al. (2003) performed the first baseline study of bivalves in the Pantai River and Sematan mangrove forest, Sarawak. After that, extensive taxonomic studies were conducted by Hamli et al. (2012b); whereas Wong \& Arshad (2011) published a significant checklist of bivalves. In a publication that reported edible bivalves and gastropods from different markets in Sarawak and that was published very recently which dealt with the morphometric and diversity investigation, we excluded that publication from our checklist due to the time span (1791-2020) in which it was published; however, the paper reported one new record Arcuatula arcuatula (Hanley, 1843) and rest of the species were already 
Table 1. Bivalve fauna in Malaysian Borneo.

\begin{tabular}{|c|c|c|c|c|c|c|}
\hline $\begin{array}{l}\text { Order/Infra Class/ } \\
\text { Super Order/ Sub } \\
\text { Class }\end{array}$ & Super Family & Family & Species & IUCN & Habitat & Ref \\
\hline \multirow{4}{*}{ Adapedonta } & \multirow{4}{*}{ Solenoidea } & Pharidae & Sinonovacula constricta (Lamarck, 1818) & NE & BW; MAR & Ashton et al. (2003) \\
\hline & & \multirow{3}{*}{ Solenidae } & $\begin{array}{l}\text { Pharella acutidens (Broderip \& Sowerby, } \\
\text { 1829) }\end{array}$ & $\mathrm{NE}$ & BW; MAR & Hamli et al. $(2012 a, b)$ \\
\hline & & & Solen lamarckii Chenu, 1843 & NE & BW; MAR & Hamli et al. $(2012 a, b)$ \\
\hline & & & Solen regularis Dunker, 1862 & $\mathrm{NE}$ & BW; MAR & Hamli et al. $(2012 a, b)$ \\
\hline \multirow{6}{*}{ Arcida } & \multirow{6}{*}{ Arcoidea } & \multirow{6}{*}{ Arcidae } & Anadara antiquata (Linnaeus, 1758) & NE & MAR & Al-Asif et al. (2020) \\
\hline & & & Anadara indica (Gmelin, 1791) & NE & MAR & Al-Asif et al. (2020) \\
\hline & & & Anadara kagoshimensis (Tokunaga, 1906) & $\mathrm{NE}$ & MAR & Al-Asif et al. (2020) \\
\hline & & & Arca ventricosa Lamarck, 1819 & NE & MAR & Wong \& Arshad (2011) \\
\hline & & & Barbatia amygdalumtostum (Röding, 1798) & NE & MAR & Wong \& Arshad (2011) \\
\hline & & & Tegillarca granosa (Linnaeus, 1758) & NE & MAR & $\begin{array}{l}\text { Hamli et al. (2012a, } \\
\text { 2012b); Shabdin et al. } \\
\text { (2014) }\end{array}$ \\
\hline \multirow{6}{*}{ Cardiida } & \multirow{3}{*}{ Cardioidea } & \multirow{3}{*}{ Cardiidae } & Tridacna crocea Lamarck, 1819 & LC & MAR & Wong \& Arshad (2011) \\
\hline & & & Tridacna maxima (Röding, 1798) & LC & MAR & Wong \& Arshad (2011) \\
\hline & & & Tridacna squamosa Lamarck, 1819 & LC & MAR & Wong \& Arshad (2011) \\
\hline & \multirow{3}{*}{ Tellinoidea } & Donacidae & Donax faba Gmelin, 1791 & NE & MAR & Al-Asif et al. (2020) \\
\hline & & Solecurtidae & Azorinus coarctatus (Gmelin, 1791) & $\mathrm{NE}$ & BW; MAR & Al-Asif et al. (2020) \\
\hline & & Tellinidae & Eurytellina lineata (W. Turton, 1819) (Pink) & NE & MAR & Al-Asif et al. (2020) \\
\hline \multirow[t]{2}{*}{ Carditida } & Carditoidea & Carditidae & Beguina semiorbiculata (Linnaeus, 1758) & NE & MAR & Wong \& Arshad (2011) \\
\hline & Crassatelloidea & Crassatellidae & Bathytormus radiatus (Sowerby, 1825) & $\mathrm{NE}$ & MAR & Al-Asif et al. (2020) \\
\hline \multirow[t]{2}{*}{ Limida } & \multirow[t]{2}{*}{ Limoidea } & \multirow[t]{2}{*}{ Limidae } & $\begin{array}{l}\text { Ctenoides philippinarum Masahito \& Habe, } \\
1978\end{array}$ & $\mathrm{NE}$ & MAR & Al-Asif et al. (2020) \\
\hline & & & Ctenoides scaber (Born, 1778) & NE & MAR & Wong \& Arshad (2011) \\
\hline Lucinida & Lucinoidea & Lucinidae & Lepidolucina venusta (Philippi, 1847) & NE & MAR & Al-Asif et al. (2020) \\
\hline \multirow{12}{*}{ Ostreida } & \multirow{6}{*}{ Ostreoidea } & \multirow{6}{*}{ Ostreidae } & Crassostrea virginica (Gmelin, 1791) & $\mathrm{NE}$ & MAR & Shabdin et al. (2014) \\
\hline & & & Lopha cristagalli (Linnaeus, 1758) & $\mathrm{NE}$ & MAR & $\begin{array}{l}\text { Shabdin et al. (2014); Wong } \\
\text { \& Arshad (2011) }\end{array}$ \\
\hline & & & Magallana bilineata (Röding, 1798) & $\mathrm{NE}$ & BW; MAR & $\begin{array}{l}\text { Shabdin (2010); Hamli et al. } \\
\text { (2012b) }\end{array}$ \\
\hline & & & Magallana rivularis (Gould, 1861) & $\mathrm{NE}$ & BW; MAR & Raven (2019) \\
\hline & & & Ostrea lurida Carpenter, 1864 & $\mathrm{NE}$ & MAR & Shabdin et al. (2014) \\
\hline & & & $\begin{array}{l}\text { Saccostrea scyphophilla (Peron \& Lesueur, } \\
1807 \text { ) }\end{array}$ & LC & MAR & Matsumoto et al. (2017) \\
\hline & \multirow{6}{*}{ Pterioidea } & \multirow{3}{*}{ Isognomonidae } & Isognomon alatus (Gmelin, 1791) & NE & MAR & Wong \& Arshad (2011) \\
\hline & & & Isognomon ephippium (Linnaeus, 1758) & NE & MAR & $\begin{array}{l}\text { Ashton et al. (2003); Hamli } \\
\text { et al. (2012b) }\end{array}$ \\
\hline & & & Isognomon nucleus (Lamarck, 1819) & NE & MAR & Matsumoto et al. (2017) \\
\hline & & Margaritidae & Pinctada margaritifera (Linnaeus, 1758) & $\mathrm{NE}$ & MAR & Wong \& Arshad (2011) \\
\hline & & Malleidae & Malleus albus Lamarck, 1819 & NE & MAR & Wong \& Arshad (2011) \\
\hline & & Pteriidae & Pteria colymbus (Röding, 1798) & NE & MAR & Wong \& Arshad (2011) \\
\hline \multirow{5}{*}{ Myida } & \multirow{5}{*}{ Pholadoidea } & \multirow{5}{*}{ Pholadidae } & Pholas orientalis Gmelin, 1791 & NE & BW; MAR & Hamli et al. $(2012 a, b)$ \\
\hline & & & $\begin{array}{l}\text { Lignopholas chengi Turner \& Santhakumaran, } \\
1989\end{array}$ & $\mathrm{NE}$ & MAR & $\begin{array}{l}\text { Turner \& Santhakumaran } \\
\text { (1989) }\end{array}$ \\
\hline & & & Lignopholas rivicola (G.B. Sowerby II, 1849) & $\mathrm{NE}$ & MAR & $\begin{array}{l}\text { Turner \& Santhakumaran } \\
\text { (1989) }\end{array}$ \\
\hline & & & Lignopholas fluminalis (Blanford, 1867) & $\mathrm{NE}$ & FW & $\begin{array}{l}\text { Turner \& Santhakumaran } \\
\text { (1989) }\end{array}$ \\
\hline & & & Martesia striata (Linnaeus, 1758) & $\mathrm{NE}$ & BW & $\begin{array}{l}\text { Turner \& Santhakumaran } \\
\text { (1989) }\end{array}$ \\
\hline \multirow{2}{*}{ Mytilida } & \multirow{2}{*}{ Mytiloidea } & \multirow{2}{*}{ Mytilidae } & Byssogerdius striatulus (Hanley, 1843) & NE & BW; MAR & Huber (2010); Raven (2019) \\
\hline & & & Brachidontes variabilis (Krauss, 1848) & $\mathrm{NE}$ & MAR & Raven (2019) \\
\hline
\end{tabular}




\begin{tabular}{|c|c|c|c|c|c|c|}
\hline $\begin{array}{l}\text { Order/Infra Class/ } \\
\text { Super Order/ Sub } \\
\text { Class }\end{array}$ & Super Family & Family & Species & IUCN & Habitat & Ref \\
\hline \multirow{7}{*}{ Pectinida } & \multirow{2}{*}{ Anomioidea } & Anomiidae & Enigmonia aenigmatica (Holten, 1802) & $\mathrm{NE}$ & BW; MAR & $\begin{array}{l}\text { Ashton et al. (2003); Raven } \\
(2019)\end{array}$ \\
\hline & & Placunidae & Placuna placenta (Linnaeus, 1758) & $\mathrm{NE}$ & BW; MAR & Hamli et al. (2012b) \\
\hline & \multirow{5}{*}{ Pectinoidea } & \multirow{2}{*}{ Spondylidae } & Spondylus gussonii O.G. Costa, 1830 & NE & MAR & Wong \& Arshad (2011) \\
\hline & & & Spondylus squamosus Schreibers, 1793 & $\mathrm{NE}$ & MAR & Wong \& Arshad (2011) \\
\hline & & \multirow{3}{*}{ Pectinidae } & Amusium pleuronectes (Linnaeus, 1758) & $\mathrm{NE}$ & MAR & Hamli et al. $(2012 a, b)$ \\
\hline & & & Mimachlamys varia (Linnaeus, 1758) & NE & MAR & Wong \& Arshad (2011) \\
\hline & & & Pedum spondyloideum (Gmelin, 1791) & $\mathrm{NE}$ & MAR & Wong \& Arshad (2011) \\
\hline \multirow{10}{*}{ Unionida } & \multirow{10}{*}{ Unionoidea } & \multirow{10}{*}{ Unionidae } & Ctenodesma borneensis (Issel, 1874) & $\mathrm{NE}$ & FW & $\begin{array}{l}\text { Zieritz \& Lopes-Lima } \\
\text { (2018); Zieritz et al. (2020) }\end{array}$ \\
\hline & & & Monodontina walpolei (Hanley, 1871) & $\mathrm{NE}$ & FW & $\begin{array}{l}\text { Zieritz \& Lopes-Lima } \\
\text { (2018); Zieritz et al. (2020) }\end{array}$ \\
\hline & & & Pilsbryoconcha exilis (I. Lea, 1838) & LC & FW & Hamli et al. (2012b) \\
\hline & & & Pressidens insularis (Drouët, 1894) & $\mathrm{NE}$ & FW & $\begin{array}{l}\text { Zieritz \& Lopes-Lima } \\
\text { (2018); Zieritz et al. (2020) }\end{array}$ \\
\hline & & & Rectidens sumatrensis (Dunker, 1852) & DD & FW & Zieritz \& Lopes-Lima (2018) \\
\hline & & & Schepmania nieuwenhuisi (Schepman, 1898) & $\mathrm{NE}$ & FW & $\begin{array}{l}\text { Zieritz \& Lopes-Lima } \\
\text { (2018); Zieritz et al. (2020) }\end{array}$ \\
\hline & & & $\begin{array}{l}\text { Schepmania parcesculpta (von Martens, } \\
\text { 1903) }\end{array}$ & $\mathrm{NE}$ & FW & $\begin{array}{l}\text { Zieritz \& Lopes-Lima } \\
\text { (2018); Zieritz et al. (2020) }\end{array}$ \\
\hline & & & Simpsonella gracilis (I. Lea, 1851) & $\mathrm{NE}$ & FW & Zieritz \& Lopes-Lima (2018) \\
\hline & & & Sinanodonta lauta (von Martens, 1877) & NE & FW & Zieritz et al. (2020) \\
\hline & & & Sinanodonta woodiana (I. Lea, 1834) & LC & FW & $\begin{array}{l}\text { Hamli et al. (2012a,b); } \\
\text { Hamli et al. (2020); Zieritz } \\
\text { \& Lopes-Lima (2018) }\end{array}$ \\
\hline \multirow{19}{*}{ Venerida } & \multirow{14}{*}{ Veneroidea } & \multirow{14}{*}{ Veneridae } & Callista erycina (Linnaeus, 1758) & $\mathrm{NE}$ & MAR & Al-Asif et al. (2020) \\
\hline & & & Circe scripta (Linnaeus, 1758) & $\mathrm{NE}$ & MAR & Hamli et al. $(2012 a, b)$ \\
\hline & & & Gafrarium pectinatum (Linnaeus, 1758) & $\mathrm{NE}$ & BW; MAR & Al-Asif et al. (2020) \\
\hline & & & Lioconcha castrensis (Linnaeus, 1758) & $\mathrm{NE}$ & MAR & Wong \& Arshad (2011) \\
\hline & & & Marcia hiantina (Lamarck, 1818) & $\mathrm{NE}$ & BW; MAR & Shabdin (2010) \\
\hline & & & Meretrix casta (Gmelin, 1791) & $\mathrm{NE}$ & BW; MAR & Al-Asif et al. (2020) \\
\hline & & & Meretrix lusoria (Röding, 1798) & NE & BW; MAR & Al-Asif et al. (2020) \\
\hline & & & Meretrix lyrata (G. B. Sowerby II, 1851) & NE & BW; MAR & $\begin{array}{l}\text { Al-Asif et al. (2020); Hamli } \\
\text { et al. (2012a,b); Hamli et } \\
\text { al. (2017) }\end{array}$ \\
\hline & & & Meretrix meretrix (Linnaeus, 1758) & $\mathrm{NE}$ & BW ; MAR & $\begin{array}{l}\text { Hamli et al. (2012a,b); Abu } \\
\text { Hena et al. (2016); Hamli et } \\
\text { al. (2016); Matsumoto et } \\
\text { al. (2017) }\end{array}$ \\
\hline & & & Paphia rotundata (Linnaeus, 1758) & NE & MAR & Wong \& Arshad (2011) \\
\hline & & & Paratapes undulatus (Born, 1778) & NE & MAR & Hamli et al. $(2012 a, b)$ \\
\hline & & & Placamen isabellina (Philippi, 1849) & $\mathrm{NE}$ & MAR & Al-Asif et al. (2020) \\
\hline & & & Pelecyora exilium (G. B. Sowerby III, 1909) & $\mathrm{NE}$ & MAR & Sowerby (1909) \\
\hline & & & Marcia japonica (J. F. Gmelin 1791) & $\mathrm{NE}$ & MAR & Gmelin (1791) \\
\hline & \multirow{4}{*}{ Cyrenoidea } & \multirow{3}{*}{ Cyrenidae } & Corbicula fluminea (O. F. Müller, 1774) & LC & FW; BW & Shabdin \& Alfred (2007) \\
\hline & & & Geloina bengalensis (Lamarck, 1818) & LC & BW & $\begin{array}{l}\text { Hamli et al. (2012a,b); } \\
\text { Hamli et al. (2015) }\end{array}$ \\
\hline & & & Geloina expansa (Mousson, 1849) & LC & BW & $\begin{array}{l}\text { Hamli et al. (2012a,b); } \\
\text { Shabdin \& Alfred (2007); } \\
\text { Shabdin (2010); Hamli et } \\
\text { al. (2015) }\end{array}$ \\
\hline & & Glauconomidae & Glauconome virens (Linnaeus, 1767) & $\mathrm{NE}$ & MAR & Hamli et al. $(2012 a, b)$ \\
\hline & Arcticoidea & Trapezidae & Neotrapezium sublaevigatum (Lamarck, 1819) & NE & MAR & Raven (2019) \\
\hline
\end{tabular}

NE-Not Evaluated | LC-Least Concern | DD-Data Deficient | FW-Freshwater | BW-Brackish water | MAR-Marine. 
available in our checklist (Idris et al. 2021). There are now a total of 19 published publications accessible, including a book, on the subject (Zieritz \& Lopes-Lima 2018). Among the published papers, 10 were published in Scopus indexed journals, the other nine in local nonindexed journals. Six published documents discuss marine bivalves, another six discuss brackish habitats; whilst the papers cover freshwater and freshwatermarine habitats.

\section{DISCUSSION}

A comprehensive checklist on Malaysian marine molluscs by Wong \& Arshad (2011) documented 581 species. Before this, Way \& Purchon (1981) and Morris \& Purchon (1981) reported 398 species (330 gastropods and 68 bivalves) from Malaysia and its adjacent coastal waters. In our study, we found bivalves from order Venerida (19 species) has the highest number of species, followed by Ostreida (12) and the freshwater order Unionida (10 species), while the rest of the orders or other entities have less than ten members. Among superfamilies, the Veneroidea (14 species) has the highest number of species, followed by the freshwater Unionoidea (10 species), and the rest of the superfamilies has less than 10 species (Figure 4). The family Veneridae comprises 14 species which is the highest among all families, following that the freshwater family Unionidae (10 species) has the second-highest number and the remaining 25 families comprise less than ten species each (Table 1).

For several recorded species it is evident the names are erroneous, as those species only occur in other continents. They are marked in the checklist (Table 2 ). The present findings suggested that some of the species were either misidentified or their introduction to Malaysian habitat might occurred; while observing their original distribution. For example, Anadara kagoshimensis (Tokunaga, 1906) is distributed in the temperate North Pacific (Zenetos et al. 2010), but the current study suggested that these species were found in the water of Malaysian Borneo (Al-Asif et al. 2020). The other distributional conflicts observed in Ctenoides scaber (Born, 1778) (Turgeon et al. 2009), Ostrea lurida Carpenter, 1864 (Polson et al. 2009), Crassostrea virginica (Gmelin, 1791) (Amaral \& Simone 2014), Isognomon alatus (Gmelin, 1791) (Tëmkin 2010), and Pteria colymbus (Röding, 1798) (Tëmkin 2010) where all known distributions of abovementioned species are either North America or South America. The European Spondylus gussonii (O.G. Costa, 1830) (Gofas et al. 2001) was also reported from Malaysian habitat, and the geographic distribution should not be in Malaysian Borneo. Although Saccostrea scyphophilla (Peron \& Lesueur, 1807) (reported as Saccostrea mordax (Gould, 1850), the materials were observed from the "Feejee Islands"(Fiji); and the species was originally described from Australia) is considered native in Australia but in 2004 the study of Lam \& Morton (2004) reported from Hong Kong coast, which might be disperse from Hong Kong to Malaysia through ocean-going ships or other means.

Additionally, some species may have been misidentified, but this cannot be determined without photographs or voucher material. In the literature we

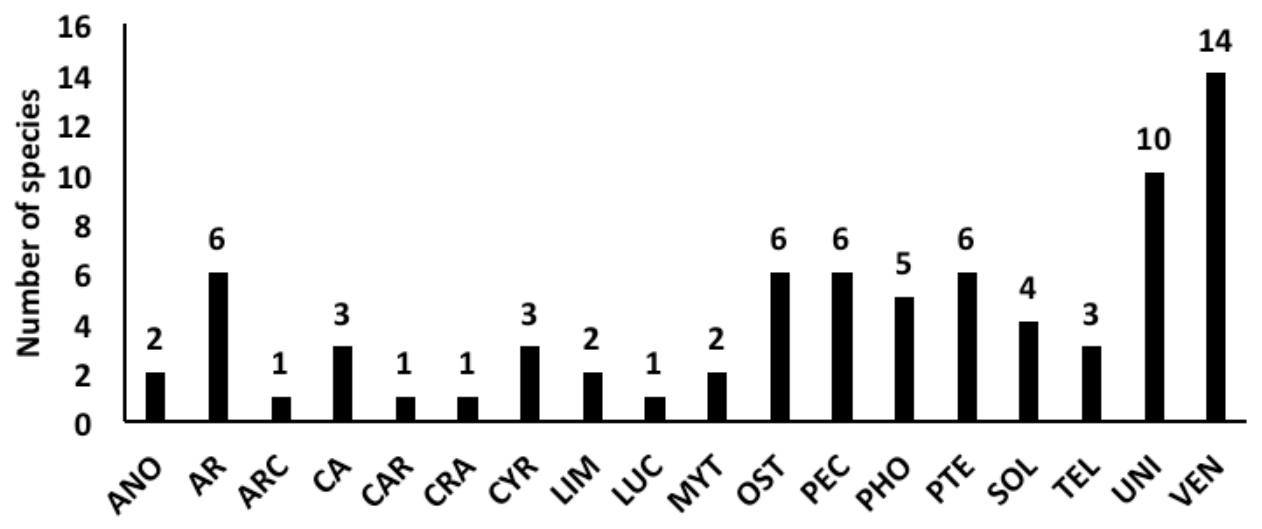

Superfamilies

Figure 4. Number of species of bivalve superfamilies recorded from Malaysian Borneo.

Ano-Anomioidea | Ar-Arcoidea | Arc-Arcticoidea | Ca-Cardioidea | Car-Carditoidea | Cra-Crassatelloidea | Cyr-Cyrenoidea | LimLimoidea | Luc-Lucinoidea | Myt-Mytiloidea | Ost-Ostreoidea | Pec-Pectinoidea | Pho-Pholadoidea | Pte-Pterioidea | Sol-Solenoidea | Tel-Tellinoidea | Uni-Unionoidea | Ven-Veneroidea. 
found many inconsistencies, while the present analysis revealed most inconsistencies were "unaccepted" according to WoRMS (2021) (i.e., the genus or species name is no longer valid); the rest were misspellings, alternative representations, changes in families, changes in author, and changes in year (Table 2). Moreover, there are taxonomic corrections: for instance, in freshwater family Unionidae there is no difference between Pseudodon crassus Drouet \& Chaper, 1892 and Pseudodon walpolei (Hanley, 1871); therefore, WoRMS merges them into one single species Monodontina walpolei (Hanley, 1871). Similarly, in the Cyrenidae Polymesoda erosa auct. non Lightfoot, 1786 and Polymesoda expansa (Mousson, 1849) have recently been synonymized in WoRMS (Huber 2010) to the revised name Geloina expansa (Mousson, 1849). The study of Hamli et al. (2015) revealed morphological differences between these two taxa which lead to considered as they were both valid species.

The current study demonstrates that current bivalve research knowledge (ecological, taxonomic, and other aspects) are insufficient to serve as a foundation for academic, conservation, and aquaculture initiatives in Malaysian Borneo. A thorough literature search was conducted using a variety of databases (e.g., SCOPUS, Web of Science, university websites (for thesis), and (NKI), but the number of published papers on Borneo bivalves was determined to be insufficient. Bivalve research in Borneo is strongly encouraged, and areas such as populations, threats, life history, and breeding biology for aquaculture initiatives can all be considered significant research fields. While taxonomy, habitat ecology, conservation actions, area-based management initiatives, and approaches to recovery and reintroduction are all fundamental, harvesting trends are also critical (Lopes-Lima et al. 2018; Zieritz et al. 2020). A comprehensive checklist of bivalves in Malaysian Borneo is necessary to fill this knowledge gap. It is recommended that additional research on bivalves be conducted as a basis for conservation measures, as they contribute to both the ecology and economy of Malaysia.

\section{Present status}

\section{IUCN status of bivalves in Borneo and their habitats}

According to the IUCN red list status, 66 bivalve species have not been evaluated by the IUCN or any other institution that are present in Malaysian Borneo, and it is quite clear that a plethora of research work can be conducted to evaluate only the IUCN unevaluated species. Whereas nine species were determined to be least concerned and one species was determined to

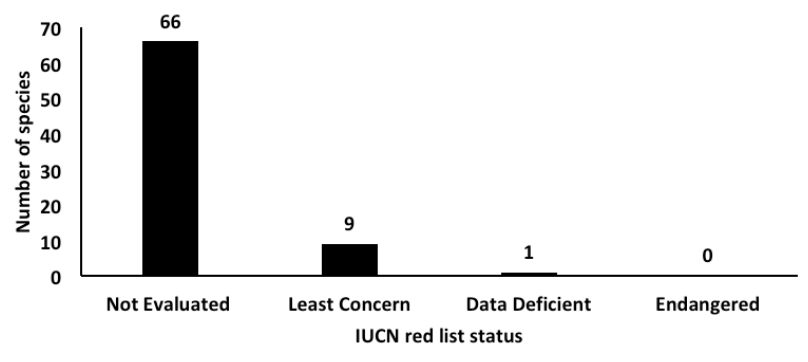

Figure 5. IUCN Red List status of bivalves in Malaysian Borneo.

have data deficiency (Figure 5). Thus, these species must be protected wherever they occur in Malaysian Borneo through the imposition of reserve areas, restricted areas, or national/regional conservation sites. It is observed that 76 species of bivalve fauna have been reported from Malaysian Borneo, including 61 marine species (17 species can be found in both marine and estuarine or brackish water), three brackish water, and 12 freshwater species (one species can be found in both fresh and brackish water).

\section{Threats to the biodiversity of bivalve species}

Sarawak and Sabah (the Malaysian portion of Borneo) are rich in biodiversity. Certain areas of Borneo Island remain pristine due to the lack of human intervention. Commercial logging and forest destruction due to palm plantations, on the other hand, have increased rapidly in various parts of these two provinces (Bryan et al. 2013; Shevade \& Loboda 2019). As a result of soil runoff into the South China Sea, secondary pollution of marine and coastal ecosystems occurs (Morni et al. 2017). Harvesting edible bivalves from wild sources indiscriminately is also a significant threat to sustainable populations. Most importantly, there is no government or local government initiative to initiate commercial aquaculture of these bivalves in order to conserve their indigenous characteristics. A model of the global declination of bivalves was proposed by Lopes-Lima et al. (2018), in which they showed that in the IndoPacific region, pollution (45\%) is the significant reason of decline in bivalve species, whilst freshwater bivalve species decline more rapidly than the marine species (Agudo-Padrón 2011). Other factors contributing to the decline of bivalve fauna include overexploitation (20\%), habitat modification (15\%), and urbanization (10\%) (Figure 6), mining activities, agriculture and aquaculture, transportation infrastructure, climate change and temperature rise, recreational activities, and various geological events, such as tsunamis caused by earthquakes. 


\section{Conservation prospects}

Conservation is critical to preventing the extinction of vulnerable species. After discussing possible causes of bivalve species decline in the Indo-Pacific region, including Malaysia, we propose some conservation strategies for sustainable use of bivalve natural bio resources based on the global model developed by Lopes-Lima et al. (2018) (Figure 8).

To begin, bivalves are aquatic Mollusca that cannot survive without water (marine, brackish, or freshwater), and thus protection of water and water-adjacent land (40\%) should be prioritized for bivalve species conservation. Additionally, awareness-raising among stakeholders (including government, the general public, universities, non-governmental organizations, and the local populace) and communication with the local populace must be implemented (25\%). Water and adjacent land management ( $12 \%)$, species management through proper conservation procedures (10\%), and incentives for local stakeholders who will carry out the conservation process ( $4 \%$ ) can all contribute significantly to the conservation of bivalves in Borneo. While the existing policies and regulations are sufficient for a sustainable conservation process, additional research is necessary to determine whether any revisions to those policies and regulations are necessary (3\%). Ex situ conservation ( $2 \%$ ) and proper enforcement of policies, legislation, and regulation ( $2 \%$ ). Any threatened species and those that have been suppressed by stressors, including human intervention, should be recovered through the application of appropriate management guidelines and procedures (1\%). Conservation strategies can be integrated into formal national curricula; consequently, future leaders and stakeholders should be concerned about bivalve biodiversity conservation $(0.5 \%)$. Reintroduction of species from another source is sometimes feasible. The general training received by common people, stakeholders, conservationists, and government officials is sufficient in the Indo-Pacific region and Malaysia, as there are ample training facilities and current conservation legislation is adequate, but conservation measures for bivalves should be prioritized.

Another research by Lopes-Lima et al. (2014) suggested that research on different aspects of taxonomy, systematics, anatomy, physiology, ecology, and conservation of freshwater bivalves will be helpful to conserve and reduce the extinction risk. Omics approach will also be helpful to conserve the bivalve fauna (Carducci et al. 2020). In contrast, a recent study from China suggested that awareness among people regarding ecological protections can be a helpful tool for

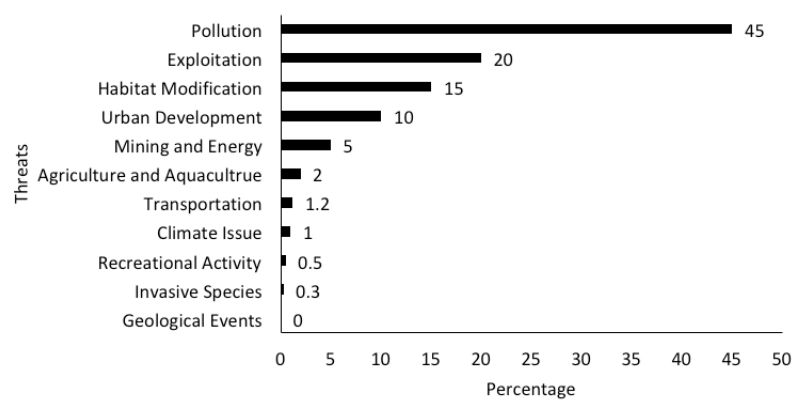

Figure 6. Reasons of bivalve decline in Malaysian Borneo (Indo-Pacific model). Adopted from Lopes-Lima et al. (2018).

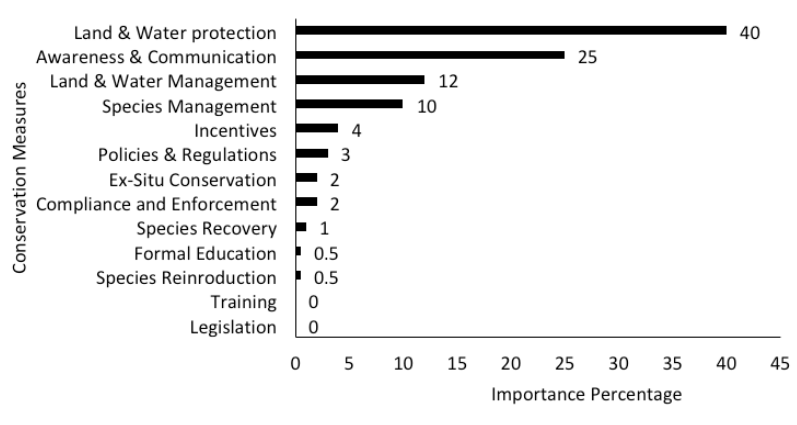

Figure 7. Conservation approach of bivalve fauna in Malaysian Borneo (Indo-Pacific Model). Adopted from Lopes-Lima et al. (2018).

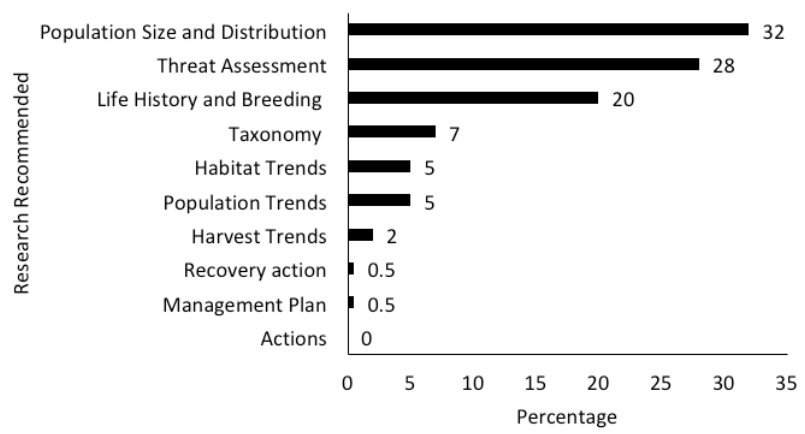

Figure 8. Recommended bivalve Research in Malaysian Borneo (IndoPacific Model). Adopted from Lopes-Lima et al. (2018).

protecting the habitat of bivalves. Reduce or suspend the commercial capture of wild bivalves, establish sanctuaries for habitat protection, extend the fishing or capture ban period which might helpful to conserve the bivalve fauna (Cao et al. 2018).

\section{Prospects and future research}

The status of bivalves in Malaysian Borneo as a whole has not yet been determined. Numerous research groups comprised of provincial governments, universities, and the federal government can work in various ecological niches to determine the true number 
Table 2. List of taxonomic corrections in available bivalve species in Malaysian Borneo.

\begin{tabular}{|c|c|c|c|c|}
\hline Given Family & Corrected Family & Given name of species & Corrected name of species & $\begin{array}{l}\text { Type of } \\
\text { inconsistency }\end{array}$ \\
\hline Arcidae & Arcidae & Anadara granosa & Tegillarca granosa (Linnaeus, 1758) & Unaccepted \\
\hline Arcidae & Arcidae & Barbatia fusca (Bruguière, 1789) & $\begin{array}{l}\text { Barbatia amygdalumtostum (Röding, } \\
\text { 1798) }\end{array}$ & Unaccepted \\
\hline Cardiidae & Cardiidae & $\begin{array}{l}\text { Tridacna (Chametrachea) crocea Lamarck, } \\
1819\end{array}$ & Tridacna crocea (Lamarck, 1819) & $\begin{array}{l}\text { Alternative } \\
\text { representation }\end{array}$ \\
\hline Cardiidae & Cardiidae & $\begin{array}{l}\text { Tridacna (Chametrachea) maxima (Röding, } \\
\text { 1798) }\end{array}$ & Tridacna maxima (Röding, 1798) & $\begin{array}{l}\text { Alternative } \\
\text { representation }\end{array}$ \\
\hline Tridacnidae & Cardiidae & Tridacna squamosa Lamarck, 1819 & Tridacna squamosa Lamarck, 1819 & Family changed \\
\hline Cyrenidae & Cyrenidae & Polymesoda bengalensis & Geloina bengalensis (Lamarck, 1818) & Unaccepted \\
\hline Cyrenidae & Cyrenidae & Polymesoda expansa & Geloina expansa (Mousson, 1849) & Unaccepted \\
\hline Glauconomidae & Glauconomidae & Gluconome virens & Glauconome virens (Linnaeus, 1767) & Misspelling \\
\hline Isognomonidae & Isognomonidae & Isonomon nucleus & Isognomon nucleus (Lamarck, 1819) & Misspelling \\
\hline Isognomonidae & Isognomonidae & Spondylus gussonii OG. Costa, 1829 & Spondylus gussonii (O.G. Costa, 1830) & Wrong year \\
\hline Limidae & Limidae & Ctenoides scabra (Born, 1778) & Ctenoides scaber (Born, 1778) & Unaccepted \\
\hline Mytilidae & Mytilidae & $\begin{array}{l}\text { Brachidontes striatulus (Hanley, } \\
\text { 1843) }\end{array}$ & Byssogerdius striatulus (Hanley, 1843) & Unaccepted \\
\hline Pteriidae & Margaritidae & Pinctada margaritifera (Linnaeus, 1758) & Pinctada margaritifera (Linnaeus, 1758) & Family changed \\
\hline Ostreidae & Ostreidae & Crassostrea virginiea & Crassostrea virginica (Gmelin, 1791) & Misspelling \\
\hline Ostreidae & Ostreidae & Crassostrea iredalei & Magallana bilineata (Röding, 1798) & Unaccepted \\
\hline Ostreidae & Ostreidae & Crassostrea rivularis (Gould, 1861) & Magallana rivularis (Gould, 1861) & $\begin{array}{l}\text { Alternative } \\
\text { representation }\end{array}$ \\
\hline Pectinidae & Pectinidae & Chlamys varia (Linnaeus, 1758) & Mimachlamys varia (Linnaeus, 1758) & $\begin{array}{l}\text { Unaccepted } \\
\text { (currently placed in } \\
\text { genus Mimachlamys) }\end{array}$ \\
\hline Myoida & Pholadidae & Pholas orientalis & Pholas orientalis (Gmelin, 1791) & Family changed \\
\hline Unionidae & Unionidae & Anodonta woodina & Sinanodonta woodiana (I. Lea, 1834) & $\begin{array}{l}\text { unaccepted } \\
\text { (recombination) }\end{array}$ \\
\hline Unionidae & Unionidae & Pseudodon walpolei (Hanley, 1871) & Monodontina walpolei (Hanley, 1871) & Unaccepted \\
\hline Veneridae & Veneridae & Meretrix. lyrata & Meretrix lyrata (G. B. Sowerby II, 1851) & Misspelling \\
\hline Veneridae & Veneridae & Paphia undulata & Paratapes undulatus (Born, 1778) & Unaccepted \\
\hline Veneridae & Veneridae & Meretrix meretrix Roding, & Meretrix meretrix (Linnaeus, 1758) & Wrong author name \\
\hline Veneridae & Veneridae & Paphia alapapilionis Röding, 1798 & Paphia rotundata (Linnaeus, 1758) & Unaccepted \\
\hline Veneridae & Veneridae & Dosinia exilium (G.B. Sowerby III, 1909) & Pelecyora exilium (G.B. Sowerby III, 1909) & Unaccepted \\
\hline Veneridae & Veneridae & Venus japonica Gmelin, 1791 & Marcia japonica (J. F. Gmelin 1791) & Unaccepted \\
\hline
\end{tabular}

and species of bivalves in Malaysian Borneo in order to create a comprehensive checklist. Aquaculture of commercially valuable bivalve species may be another area of research that could help prevent indiscriminate harvesting of bivalves from Malaysian Borneo's diverse habitats. Pollution studies can be conducted to assess the biodiversity and ecological threats posed by various industrial zones, despite the fact that water, air, and soil pollution are increasing as a result of these two provinces' rapid industrialization. A strong legislative framework could be established and enforced to protect different habitats' ecological integrity and bivalve diversity. Strict enforcement of laws may aid in the conservation of bivalve species in Malaysian Borneo.
Regrettably, there is far too little information at the moment, but provincial governments could declare some species vulnerable and also establish some protected zones in accordance with the IUCN Red List. Numerous awareness campaigns, including posters, television programmes, telecasts, documentaries, films, and cartoons, can be produced to educate the public about the critical nature of bivalve conservation. For example, state governments can take steps similar to the Chinese Giant Panda conservation approach, which is called 'Panda Diplomacy' (Buckingham et al. 2013), where China showed public awareness and scientific efforts are effective in the conservation process. Lopes-Lima et al. (2018) proposed some research aspects that will help 
retain the bivalve diversity's sustainability. According to them, the primary focus on bivalve research should be on the assessment of populations and their distribution (32 $\%)$, assessment of threats ( $28 \%$ ), and on studying their life history / breeding for future aquaculture purposes (20\%) (Figure 7). Whereas the taxonomy of specific bivalve species, the habitats and ecology of each species, the population trends of bivalves in Borneo, the harvest trends of fishers including aquaculture, recovery actions if any species faces imminent extinction, management plans for multi-ground stakeholders, and further action by various organizations can be considered as significant research arenas.

Kumar \& Ravinesh (2016) recommended that the importance of taxonomic research be disseminated; thus, taxonomic knowledge can be included in national level curricula, for example, high school and college students can learn this science with joy. This initiative can be incorporated into the provincial and regional curricula of Malaysian Borneo. Additionally, they emphasized the importance of establishing accurate species databases and repositories, which will aid in future research and analysis. Kumar \& Ravinesh (2016) also emphasized the resolution of scientifically dubious name categories, such as 'taxon inquirendum' and 'nomen dubium', which is commendable, and the protocols may be beneficial for the Malaysian Borneo ecosystem as well. They proposed that an integrative taxonomic approach incorporating detailed biogeography and evolutionary genetic materials could be beneficial for bivalve fauna conservation in Malaysian Borneo. Finally, citizen scientists and civil society approaches are very common and widely adopted in many developed countries; for this, a person does not have to be a scientist; rather, a keen interest in nature and biodiversity can also be beneficial for nature conservation. The research on the aforementioned criteria may be adopted and contribute to the conservation of biodiversity in Malaysian Borneo in the coming years and decades.

The current checklist is prepared by reviewing the previously published documents from Malaysian Borneo, although the published documents are few. Some of the papers we had collected were very general, and the author did not provide an appropriate format of species scientific names (Al-Asif et al. 2020). Misidentification is a widespread issue in taxonomy, and some published documents reported different bivalve species out ranged of their original distribution region. For example, the distribution of Ctenoides scaber (Born, 1778) (Turgeon et al. 2009) is well known from North America, but the previous study reported this species from the southeastern Asian region. This might happen because the author found similarities with southeastern Asian bivalve species with North American species or is entirely misidentified.

On the other hand, we can say it is considered either misidentification of these species or they introduced to the Malaysian habitat. Most of the published papers we had handled did not provide any pictures of bivalve species, which can be considered a considerable gap of the bivalve research in Malaysian Borneo (Shabdin 2010). In contrast, the papers that were published on the ecological phenomena or on the ecological subject matter did not include photographs or appropriate scientific nomenclature, and the samples that had been gathered were not stored in a permanent and easily accessible repository for future study. The island of Borneo does not have a natural history museum, although there is a tiny part of the 'Sarawak State Museum' that is known as the 'Natural History Museum', but there are no depositing facilities or a permanent repository in the Malaysian part of this island (Al-Asif et al. 2020; Shabdin 2010). Given the foregoing, Malaysian Borneo urgently requires a permanent and accessible repository for the collection of samples. New expeditions to different rivers and creeks in the interior of Borneo can be conducted to determine the exact number of bivalve species found in Malaysian Borneo.

\section{CONCLUSIONS}

The current work produced a comprehensive checklist of bivalves recorded from Malaysian Borneo, crosschecked with WoRMS (2021) and MolluscaBase (2021). An accurate checklist of bivalves aids appropriate resource allocation for the conservation process, and at the same time has many other functions. Accurate data on bivalve species under one umbrella will provide insight which species are present in Malaysian Borneo. It will also help revise and update the national list of molluscan fauna and periodic update of bivalve taxonomic information.

\section{REFERENCES}

Abu Hena, M.K., M.H. Idris, R.M.Y. Khairul, M.K.A. Bhuiyan, N. Hoque \& U. Kumar (2016). Diversity of macro-benthos in the mangrove forest of Kuala Sibuti, Miri, Sarawak. In: Malaysia International Biology Symposium (i-SIMBIOMAS 2016). Malaysia International Biology Symposium, Putrajaya International Convention Center, Putrajaya, Malaysia.

Agudo-Padrón, I.A. (2011). Threatened freshwater and terrestrial 
molluscs (Mollusca, Gastropoda et Bivalvia) of Santa Catarina state, Southern Brazil: check list and evaluation of regional threats. Biodiversity Journal 2(2): 59-66.

Al-Asif, A.A., H. Hamli, M.K. Abu Hena, M.H. Idris, G.J. Gerusu, J.B. Ismail \& N.U. Karim (2020). Benthic macrofaunal assemblage in seagrass-mangrove complex and adjacent ecosystems of PunangSari estuary, Lawas, Sarawak, Malaysia. Biodiversitas 21(10): 46064615. https://doi.org/10.13057/biodiv/d211019

Alam, R., S. Sharmin, S.M. Majharul \& A. Alam (2020). Salinity intrusion affects early development of freshwater aquaculture species pabda, Ompok pabda. Aquaculture Reports 18: 100476. https://doi.org/10.1016/j.aqrep.2020.100476

Amano, T. \& W.J. Sutherland (2013). Four barriers to the global understanding of biodiversity conservation: Wealth, language geographical location and security. Proceedings of the Roya Society B: Biological Sciences 280(1756). https://doi.org/10.1098/ rspb.2012.2649

Amaral, V.S.D. \& L.R.L. Simone (2014). Revision of genus Crassostrea (Bivalvia: Ostreidae) of Brazil. Journal of the Marine Biological Association of the United Kingdom 94(4): 811-836. https://doi. org/10.1017/S0025315414000058

Ashton, E.C., D.J. Macintosh \& P.J. Hogarth (2003). A baseline study of the diversity and community ecology of crab and molluscan macrofauna in the Sematan mangrove forest, Sarawak, Malaysia. Journal of Tropical Ecology 19(2): 127-142. https://doi.org/10.1017/ S0266467403003158

Braje, T.J. \& J.M. Erlandson (2013). Human acceleration of animal and plant extinctions: A late pleistocene, holocene, and anthropocene continuum. Anthropocene 4: 14-23. https://doi.org/10.1016/j. ancene.2013.08.003

Bryan, J.E., P.L. Shearman, G.P. Asner, D.E. Knapp, G. Aoro \& B. Lokes (2013). Extreme differences in forest degradation in Borneo: Comparing practices in Sarawak, Sabah, and Brunei. PLoS ONE 8(7): e69679. https://doi.org/10.1371/journal.pone.0069679

Buckingham, K.C., J.N.W. David \& P. Jepson (2013). Environmental reviews and case studies: Diplomats and refugees: Panda diplomacy, soft cuddly power, and the new trajectory in panda conservation. Environmental Practice 15(3): 262-270. https://doi.org/10.1017/ S1466046613000185

Cao, Y.L., X.J. Liu, R.W. Wu, T. Xue, L. Li, C.H. Zhou, S. Ouyang \& X.P. Wu (2018). Conservation of the endangered freshwater mussel Solenaia carinata (Bivalvia, Unionidae) in China. Nature Conservation 26: $33-$ 53. https://doi.org/10.3897/natureconservation.26.25334

Carducci, F., M.A. Biscotti, E. Trucchi, M.E. Giuliani, S. Gorbi, A. Coluccelli, M. Barucca \& A. Canapa (2020). Omics approaches for conservation biology research on the bivalve Chamelea gallina Scientific Reports 10(1): 1-15. https://doi.org/10.1038/s41598-02075984-9

Chaklader, R., M. Abu, B. Siddik, A. Hanif, A. Nahar, S. Mahmud \& M. Piria (2016). Morphometric and meristic variation of endangered pabda catfish, Ompok pabda (Hamilton-Buchanan, 1822) from Southern coastal waters of Bangladesh. Pakistan Journal of Zoology 48(3): 681-687.

Chakraborty, B.K., Z.A. Mirza \& A. Chakraborty (2010). Reproductive cycle of the endangered Pabda, Ompok pabda (HamiltonBouchanan, 1822) in Bangladesh. Asian Fisheries Science 23(3): 301-320. https://doi.org/10.33997/j.afs.2010.23.3.003

Collen, B., L. Mcrae, S. Deinet, A. de Palma, T. Carranza, N. Cooper, J. Loh \& J.E.M. Baillie (2011). Predicting how populations decline to extinction. Philosophical Transactions of the Royal Society B: Biological Sciences 366(1577): 2577-2586. https://doi.org/10.1098/ rstb.2011.0015

Gmelin J.F. (1791). Vermes, pp. 3021-3910. In: Gmelin J.F. (eds.). Caroli a Linnaei Systema Naturae per Regna Tria Naturae. Ed. 13. Tome 1(6). G.E. Beer, Lipsiae [Leipzig].

Gofas, S., J. Le Renard \& P. Bouchet (2001). Mollusca: European Register of Marine Species: a check-list of the marine species in Europe and a bibliography of guides to their identification. Patrimoines Naturels 50: 180-213.
Groves, C.R., D.B. Jensen, L.L. Valutis, K.H. Redford, M.L. Shaffer, J.M. Scott, J.V. Baumgartner, J.V. Higgins, M.W. Beck \& M.G. Anderson (2002). Planning for biodiversity conservation: putting conservation science into practice. BioScience 52(6): 499-512. https://doi. org/10.1641/0006-3568(2002)052[0499:PFBCPC]2.0.CO;2

Hamli, H., A.A. Rahim, M.H. Idris, M.K.A. Hena \& W.S. King (2015). Morphometric variation among three local mangrove clam species of Corbiculidae. Songklanakarin Journal of Science and Technology 37(1): 15-20.

Hamli, H., M.H. Idris, M.K.A. Hena, A. Rajaee \& A. Arshad (2016). Inner shell as variation key of local hard clam Meretrix spp. Journal of Environmental Biologylogy 37(Special): 641-646.

Hamli, H., M.H. Idris, M.K.A. Hena \& S.K. Wong (2012a). Diversity of edible mollusc (Gastropoda and Bivalvia) at selected divison of Sarawak, Malaysia. International Journal on Advanced Science, Engineering and Information Technology 2(4): 5-7. https://doi. org/10.18517/ijaseit.2.4.202

Hamli, H., M.H. Idris, A.H. Rajaee, M.K.A. Hena \& M.N. Hoque (2017). Condition index of Meretrix lyrata (Sowerby 1851) and its relationship with water parameter in Sarawak. Sains Malaysiana 46(4): 545-551. https://doi.org/10.17576/jsm-2017-4604-05

Hamli, H., M.H. Idris, M.K.A. Hena \& S.K. Wong (2012b). Taxonomic study of edible bivalve from selected division of Sarawak, Malaysia. International Journal of Zoological Research 8(1): 5258.

Hamli, H., N. Hashim \& A. Al-Asif (2020). Isolation and potential culture of phytoplankton live feed for freshwater mussels Sinanodonta woodiana (Lea, 1834). Asian Journal of Animal Sciences 14(4): 127136. https://doi.org/10.3923/ajas.2020.127.136

Huber, M. (2010). Compendium of Bivalves. A Full-color Guide to 3,300 of the World's Marine Bivalves. A Status on Bivalvia after 250 Years of Research. ConchBooks, Hackenheim, 901 pp.

Huber, M. (2015). Compendium of Bivalves 2. A Full-color Guide to the Remaining Seven Families. A Systematic Listing of 8,500 Bivalve Species and 10,500 Synonyms. ConchBooks, Harxheim, 907 pp.

Idris, M.H., A. Arshad, S.M.N.N. Amin, S.B. Japar, S.K. Daud, A.G. Mazlan, M.S. Zakaria \& F.M. Yusoff (2012). Age, growth and lengthweight relationships of Pinna bicolor Gmelin (Bivalvia: Pinnidae) in the seagrass beds of Sungai Pulai Estuary, Johor, Peninsular Malaysia. Journal of Applied Ichthyology 28(4): 597-600. https:// doi.org/10.1111/j.1439-0426.2011.01807.x

Idris, M.H., H. Hamli, M.K.A. Hena, R.A. Lah \& N.M.S.N. Jaafar (2021). Study of diversity and morphometry in edible bivalves and gastropods from a coastal wetland in Sarawak. Songklanakarin Journal of Science and Technology 43(3): 889-896.

Işik, K. (2011). Rare and endemic species: Why are they prone to extinction? Turkish Journal of Botany 35(4): 411-417. https://doi. org/10.3906/bot-1012-90

IUCN Bangladesh (2015). Red List of Bangladesh Volume 5: Freshwater Fishes. International Union for Conservation of Nature, Bangladesh Country Office, Dhaka, Bangladesh, 360 pp.

Jasin, B. (2015). Posidonomya ( Bivalvia ) from northwest peninsular Malaysia and its significance. Sains Malaysiana 44(2): 217-223.

Kabir, M.S.U., F. Arefin, M.M. Rahman, M.R.I. Sarder \& M.F.A. Mollah (2018). Domestication of red fin mahseer (Tor tor) with supplementary feeds in captive condition in Bangladesh. Journal of the Bangladesh Agricultural University 16(3): 533-538. https://doi. org/10.3329/jbau.v16i3.39451

Köhler, F., M. Seddon, A.E. Bogan, D.V. Tu, P. Sri-Aroon \& D. Allen (2012). The status and distribution of freshwater molluscs of the Indo-Burma region pp. 66-89. In: Allen, D.J., K.G. Smith \& W.R.T. Darwall (Eds.). The Status and Distribution of Freshwater Biodiversity in Indo-Burma. IUCN, Gland, Switzerland and Cambridge, UK.

Kumar, B. \& R. Ravinesh (2016). Taxonomy of marine molluscs of India: status and challenges ahead, pp. 67-88. In: Bijoy Nandan, P., G. Oliver, P.R. Jayachandran \& C.V. Asha (Eds.) Training manual -1st International training workshop on taxonomy of bivalve molluscs. Cochin University of Science and Technology, Kochi. https://www. researchgate.net/publication/303333869_Taxonomy_of_Marine_ Molluscs_of_India_Status_and_Challenges_Ahead. 
Lam, K. \& B. Morton (2004). The oysters of Hong Kong (Bivalvia: Ostreidae and Gryphaeidae). Raffles Bulletin of Zoology 52(1): 1128.

Lee, C.H., D.K. Lee, M.A. Ali \& P.J. Kim (2008). Effects of oyster shell on soil chemical and biological properties and cabbage productivity as a liming materials. Waste Management 28(12): 2702-2708. https:// doi.org/10.1016/j.wasman.2007.12.005

Lopes-Lima, M., A. Teixeira, E. Froufe, A. Lopes, S. Varandas \& R. Sousa (2014). Biology and conservation of freshwater bivalves: Past, present and future perspectives. Hydrobiologia 735(1): 1-13. https://doi.org/10.1007/s10750-014-1902-9

Lopes-Lima, M., L.E. Burlakova, A.Y. Karatayev, K. Mehler, M. Seddon \& R. Sousa (2018). Conservation of freshwater bivalves at the global scale: Diversity, threats and research needs. Hydrobiologia 810(1): 1-14. https://doi.org/10.1007/s10750-017-3486-7

Martin, J.L., V. Maris \& D.S. Simberloff (2016). The need to respect nature and its limits challenges society and conservation science. Proceedings of the National Academy of Sciences of the United States of America 113(22): 6105-6112. https://doi.org/10.1073/ pnas. 1525003113

Matsumoto, M.B., E. Saleh, Z. Waheed, S. Muhammad Ali \& J. Madin (2017). Marine profiling of Marudu Bay, Sabah, Malaysia: Final Report. Sabah, 88 pp.

MolluscaBase (2021). Bivalvia. http://molluscabase.org/aphia. php?p=taxdetails\&id=105 on 2021-08-10

Morni, W.Z.W., S.A.K.A. Rahim, T. Masron, R. Rumpet, J. Musel \& R. Hassan (2017). Continental shelf sediments of Sarawak, Malaysian Borneo. Scientific World Journal 2017(4853048): 1-10. https://doi org/10.1155/2017/4853048

Morris, S. \& R.D. Purchon (1981). The marine shelled mollusca of West Malaysia and Singapore part 3, Bivalvia. Journal of Molluscan Studies 47(2264): 322-327. https://doi.org/10.1093/oxfordjournals.mollus. a065575

National Research Council (1992). Conserving Biodiversity: A Research Agenda for Development Agencies. The National Academies Press, Washington, DC, $138 \mathrm{pp}$.

Parvez, I., M.A. Alam, M.M. Hassan, Y. Ara, I. Hoshan \& A.S.M. Kibria (2019). A checklist of fish species from three rivers in northwestern Bangladesh based on a seven-year survey. Journal of Threatened Taxa 11(6): 13786-13794. https://doi.org/10.11609/ jott.4303.11.6.13786-13794

Polson, M.P., W.E. Hewson, D.J. Eernisse, P.K. Baker \& D.C. Zacherl (2009). You say conchaphila, I say lurida: Molecular evidence for restricting the Olympia Oyster (Ostrea lurida Carpenter 1864) to temperate western North America. Journal of Shellfish Research 28(1): 11-21. https://doi.org/10.2983/035.028.0102

Raven, J.G.M. (2019). Notes on molluscs from N.W. Borneo - dispersal of molluscs through nipa rafts. The Festivus 51(1): 3-10.

Reydon, T.A.C. (2019). Are species good units for biodiversity studies and conservation efforts? p. 452. In: Wolfe, C.T., P. Huneman \& T.A.C. Reydon (Eds.). From Assessing to Conserving Biodiversity: Conceptual and Practical Challenges. Spinger.

Shabdin, M.L. (2010). Macrofauna of Rajang River, Sarawak, Malaysian Borneo. Journal of Tropical Biology and Conservation 7(1): 11-30.

Shabdin, M.L. \& A. Alfred (2007). Commercial molluscs distribution of the western part of Sarawak. The Sarawak Museum Journal 63: 167-178.

Shabdin, M.L., A. Azizil, F. Abg, S. Atiqah \& A. Rahim (2014). Marine gastropod and bivalves of Sampadi Island, Lundu, Sarawak, pp. 7587. In: Proceedings of Aquatic Science Colloquium on Pulau Sampadi Marine Life Expedition. Department of Aquatic Science, Faculty of Resource Science and Technology, Universiti Malaysia Sarawak, Sarawak.

Shevade, V.S. \& T.V. Loboda (2019). Oil palm plantations in Peninsular Malaysia: Determinants and constraints on expansion. PLOS ONE 14(2): e0210628. https://doi.org/10.1371/journal.pone.0210628
Sowerby, G.B. III. (1909). Descriptions of new species of Terebra, Pleurotoma, Trochus, Tellina, Dosina, and Modiola. Proceedings of the Malacological Society of London 8(4): 198-201.

Olivier, A.V.D.S., L. Jones, L.L. Vay, M. Christie, J. Wilson \& S.K. Malham (2020). A global review of the ecosystem services provided by bivalve aquaculture. Reviews in Aquaculture 12(1): 3-25. https:// doi.org/10.1111/raq.12301

Tëmkin, I. (2010). Molecular phylogeny of pearl oysters and their relatives (Mollusca, Bivalvia, Pterioidea). BMC Evolutionary Biology 10(1): 342. https://doi.org/10.1186/1471-2148-10-342

Turgeon, D.D., W.G. Lyons, P. Mikkelsen, G. Rosenberg \& F. Moretzsohn (2009). Bivalvia (Mollusca) of the Gulf of Mexico pp. 711-744. In: Felder, D.L. \& D.K. Camp (Eds.). Gulf of Mexico-Origins, Waters, and Biota. Biodiversity. Texas A\&M Press, Colleg.

Turner, R.D. \& L.N. Santhakumaran (1989). The genera Martesia and Lignopholas in the indo-pacific (Mollusca: Bivalvia: Pholadidae). Ophelia 30(3): 155-186. https://doi.org/10.1080/00785326.1989. 10430842

Vaughn, C.C. \& T.J. Hoellein (2018). Bivalve impacts in freshwater and marine ecosystems. Annual Review of Ecology, Evolution, and Systematics 49: 183-208. https://doi.org/10.1146/annurevecolsys-110617-062703

Venugopal, V. \& K. Gopakumar (2017). Shellfish: Nutritive value, health benefits, and consumer safety. Comprehensive Reviews in Food Science and Food Safety 16(6): 1219-1242. https://doi. org/10.1111/1541-4337.12312

Way, K. \& R.D. Purchon (1981). The marine shelled mollusca of West Malaysia and Singapore Part 3, Bivalvia. Journal of Molluscan Studies (47): 313-321. https://doi.org/10.1093/oxfordjournals. mollus.a065575

Wijsman, J.W.M., K. Troost, J. Fang \& A. Roncarati (2019). Global production of marine bivalves. Trends and challenges pp. 1-26. In: Smaal, A., J. Ferreira, J. Grant, J. Petersen \& $\varnothing$. Strand (Eds.). Goods and Services of Marine Bivalves. Springer, Cham.

Wong, N.L.W.S. \& A. Arshad (2011). A brief review on marine shelled mollusca (Gastropoda and Bivalvia) record in Malaysia. Journal of Fisheries and Aquatic Science 6(7): 669-699. https://doi. org/10.3923/jfas.2011.669.699

WoRMS (2021). Bivalvia. http://www.marinespecies.org/aphia. php?p=taxdetails\&id=105 on 2021-08-10

Zenetos, A., S. Gofas, M. Verlaque, M.E. Cinar, J.E. Garcia Raso, C.N. Bianchi, C. Morri, E. Azzurro, M. Bilecenoglu, C. Froglia, I. Siokou, D. Violanti, A. Sfriso, G. San Martin, A. Giangrande, T. Katagan, E. Ballesteros, A.A. Ramos-Espla, F. Mastrototaro, O. Ocana, A. Zingone, M.C. Gambi \& N. Streftaris (2010). Alien species in the Mediterranean Sea by 2010. A contribution to the application of European Union's Marine Strategy Framework Directive (MSFD). Part I. Spatial distribution. Mediterranean Marine Science 11(2): 381-493. https://doi.org/10.12681/mms.87

Zieritz, A. \& M. Lopes-Lima (2018). Handbook and National Red-List of the Freshwater Mussels of Malaysia. IUCN, Kuala Lumpur, 1-29pp.

Zieritz, A., M. Lopes-Lima, A.E. Bogan, R. Sousa, S. Walton, K.A.A. Rahim, J.J. Wilson, P.Y. Ng, E. Froufe \& S. McGowan (2016). Factors driving changes in freshwater mussel (Bivalvia, Unionida) diversity and distribution in Peninsular Malaysia. Science of the Total Environment 571: 1069-1078. https://doi.org/10.1016/j. scitotenv.2016.07.098

Zieritz, A., H.Taha, M. Lopes-Lima, J. Pfeiffer, K.W. Sing, Z. Sulaiman, S. McGowan \& K.A. Khairul (2020). Towards the conservation of Borneo's freshwater mussels: rediscovery of the endemic Ctenodesma borneensis and first record of the non-native Sinanodonta lauta. Biodiversity and Conservation 29(7): 2235-2253. https://doi.org/10.1007/s10531-020-01971-1

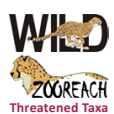



Dr. Albert G. Orr, Griffith University, Nathan, Australia

Dr. Sameer Padhye, Katholieke Universiteit Leuven, Belgium

Dr. Nancy van der Poorten, Toronto, Canada

Dr. Kareen Schnabel, NIWA, Wellington, New Zealand

Dr. R.M. Sharma, (Retd.) Scientist, Zoological Survey of India, Pune, India

Dr. Manju Siliwal, WILD, Coimbatore, Tamil Nadu, India

Dr. G.P. Sinha, Botanical Survey of India, Allahabad, India

Dr. K.A. Subramanian, Zoological Survey of India, New Alipore, Kolkata, India

Dr. P.M. Sureshan, Zoological Survey of India, Kozhikode, Kerala, India

Dr. R. Varatharajan, Manipur University, Imphal, Manipur, India

Dr. Eduard Vives, Museu de Ciències Naturals de Barcelona, Terrassa, Spain

Dr. James Young, Hong Kong Lepidopterists' Society, Hong Kong

Dr. R. Sundararaj, Institute of Wood Science \& Technology, Bengaluru, India

Dr. M. Nithyanandan, Environmental Department, La Ala Al Kuwait Real Estate. Co. K.S.C., Kuwait

Dr. Himender Bharti, Punjabi University, Punjab, India

Mr. Purnendu Roy, London, UK

Dr. Saito Motoki, The Butterfly Society of Japan, Tokyo, Japan

Dr. Sanjay Sondhi, TITLI TRUST, Kalpavriksh, Dehradun, India

Dr. Nguyen Thi Phuong Lien, Vietnam Academy of Science and Technology, Hanoi, Vietnam

Dr. Nitin Kulkarni, Tropical Research Institute, Jabalpur, India

Dr. Robin Wen Jiang Ngiam, National Parks Board, Singapore

Dr. Lional Monod, Natural History Museum of Geneva, Genève, Switzerland.

Dr. Asheesh Shivam, Nehru Gram Bharti University, Allahabad, India

Dr. Rosana Moreira da Rocha, Universidade Federal do Paraná, Curitiba, Brasil

Dr. Kurt R. Arnold, North Dakota State University, Saxony, Germany

Dr. James M. Carpenter, American Museum of Natural History, New York, USA

Dr. David M. Claborn, Missouri State University, Springfield, USA

Dr. Kareen Schnabel, Marine Biologist, Wellington, New Zealand

Dr. Amazonas Chagas Júnior, Universidade Federal de Mato Grosso, Cuiabá, Brasil

Mr. Monsoon Jyoti Gogoi, Assam University, Silchar, Assam, India

Dr. Heo Chong Chin, Universiti Teknologi MARA (UiTM), Selangor, Malaysia

Dr. R.J. Shiel, University of Adelaide, SA 5005, Australia

Dr. Siddharth Kulkarni, The George Washington University, Washington, USA

Dr. Priyadarsanan Dharma Rajan, ATREE, Bengaluru, India

Dr. Phil Alderslade, CSIRO Marine And Atmospheric Research, Hobart, Australia

Dr. John E.N. Veron, Coral Reef Research, Townsville, Australia

Dr. Daniel Whitmore, State Museum of Natural History Stuttgart, Rosenstein, Germany.

Dr. Yu-Feng Hsu, National Taiwan Normal University, Taipei City, Taiwan

Dr. Keith V. Wolfe, Antioch, California, USA

Dr. Siddharth Kulkarni, The Hormiga Lab, The George Washington University, Washington,

D.C., USA

Dr. Tomas Ditrich, Faculty of Education, University of South Bohemia in Ceske

Budejovice, Czech Republic

Dr. Mihaly Foldvari, Natural History Museum, University of Oslo, Norway

Dr. V.P. Uniyal, Wildlife Institute of India, Dehradun, Uttarakhand 248001, India

Dr. John T.D. Caleb, Zoological Survey of India, Kolkata, West Bengal, India

Dr. Priyadarsanan Dharma Rajan, Ashoka Trust for Research in Ecology and the Environment (ATREE), Royal Enclave, Bangalore, Karnataka, India

\section{Fishes}

Dr. Neelesh Dahanukar, IISER, Pune, Maharashtra, India

Dr. Topiltzin Contreras MacBeath, Universidad Autónoma del estado de Morelos, México

Dr. Heok Hee Ng, National University of Singapore, Science Drive, Singapore

Dr. Rajeev Raghavan, St. Albert's College, Kochi, Kerala, India

Dr. Robert D. Sluka, Chiltern Gateway Project, A Rocha UK, Southall, Middlesex, UK

Dr. E. Vivekanandan, Central Marine Fisheries Research Institute, Chennai, India

Dr. Davor Zanella, University of Zagreb, Zagreb, Croatia

Dr. A. Biju Kumar, University of Kerala, Thiruvananthapuram, Kerala, India

Dr. Akhilesh K.V., ICAR-Central Marine Fisheries Research Institute, Mumbai Research

Centre, Mumbai, Maharashtra, India

Dr. J.A. Johnson, Wildlife Institute of India, Dehradun, Uttarakhand, India

\section{Amphibians}

Dr. Sushil K. Dutta, Indian Institute of Science, Bengaluru, Karnataka, India

Dr. Annemarie Ohler, Muséum national d'Histoire naturelle, Paris, France

\section{Reptiles}

Dr. Gernot Vogel, Heidelberg, Germany

Dr. Raju Vyas, Vadodara, Gujarat, India

Dr. Pritpal S. Soorae, Environment Agency, Abu Dubai, UAE.

Prof. Dr. Wayne J. Fuller, Near East University, Mersin, Turkey

Prof. Chandrashekher U. Rivonker, Goa University, Taleigao Plateau, Goa. India

Dr. S.R. Ganesh, Chennai Snake Park, Chennai, Tamil Nadu, India

Dr. Himansu Sekhar Das, Terrestrial \& Marine Biodiversity, Abu Dhabi, UAE
Birds

Dr. Hem Sagar Baral, Charles Sturt University, NSW Australia

Dr. Chris Bowden, Royal Society for the Protection of Birds, Sandy, UK

Dr. Priya Davidar, Pondicherry University, Kalapet, Puducherry, India

Dr. J.W. Duckworth, IUCN SSC, Bath, UK

Dr. Rajah Jayapal, SACON, Coimbatore, Tamil Nadu, India

Dr. Rajiv S. Kalsi, M.L.N. College, Yamuna Nagar, Haryana, India

Dr. V. Santharam, Rishi Valley Education Centre, Chittoor Dt., Andhra Pradesh, India

Dr. S. Balachandran, Bombay Natural History Society, Mumbai, India

Mr. J. Praveen, Bengaluru, India

Dr. C. Srinivasulu, Osmania University, Hyderabad, India

Dr. K.S. Gopi Sundar, International Crane Foundation, Baraboo, USA

Dr. Gombobaatar Sundev, Professor of Ornithology, Ulaanbaatar, Mongolia

Prof. Reuven Yosef, International Birding \& Research Centre, Eilat, Israel

Dr. Taej Mundkur, Wetlands International, Wageningen, The Netherlands

Dr. Carol Inskipp, Bishop Auckland Co., Durham, UK

Dr. Tim Inskipp, Bishop Auckland Co, Durham, UK

Dr. V. Gokula, National College, Tiruchirappalli, Tamil Nadu, India

Dr. Arkady Lelej, Russian Academy of Sciences, Vladivostok, Russia

Dr. Simon Dowell, Science Director, Chester Zoo, UK

Dr. Mário Gabriel Santiago dos Santos, Universidade de Trás-os-Montes e Alto Douro,

Quinta de Prados, Vila Real, Portugal

Dr. Grant Connette, Smithsonian Institution, Royal, VA, USA

Dr. M. Zafar-ul Islam, Prince Saud Al Faisal Wildlife Research Center, Taif, Saudi Arabia

Mammals

Dr. Giovanni Amori, CNR - Institute of Ecosystem Studies, Rome, Italy

Dr. Anwaruddin Chowdhury, Guwahati, India

Dr. David Mallon, Zoological Society of London, UK

Dr. Shomita Mukherjee, SACON, Coimbatore, Tamil Nadu, India

Dr. Angie Appel, Wild Cat Network, Germany

Dr. P.O. Nameer, Kerala Agricultural University, Thrissur, Kerala, India

Dr. Ian Redmond, UNEP Convention on Migratory Species, Lansdown, UK

Dr. Heidi S. Riddle, Riddle's Elephant and Wildlife Sanctuary, Arkansas, USA

Dr. Karin Schwartz, George Mason University, Fairfax, Virginia.

Dr. Lala A.K. Singh, Bhubaneswar, Orissa, India

Dr. Mewa Singh, Mysore University, Mysore, India

Dr. Paul Racey, University of Exeter, Devon, UK

Dr. Honnavalli N. Kumara, SACON, Anaikatty P.O., Coimbatore, Tamil Nadu, India

Dr. Nishith Dharaiya, HNG University, Patan, Gujarat, India

Dr. Spartaco Gippoliti, Socio Onorario Società Italiana per la Storia della Fauna "Giuseppe

Altobello", Rome, Italy

Dr. Justus Joshua, Green Future Foundation, Tiruchirapalli, Tamil Nadu, India

Dr. H. Raghuram, The American College, Madurai, Tamil Nadu, India

Dr. Paul Bates, Harison Institute, Kent, UK

Dr. Jim Sanderson, Small Wild Cat Conservation Foundation, Hartford, USA

Dr. Dan Challender, University of Kent, Canterbury, UK

Dr. David Mallon, Manchester Metropolitan University, Derbyshire, UK

Dr. Brian L. Cypher, California State University-Stanislaus, Bakersfield, CA

Dr. S.S. Talmale, Zoological Survey of India, Pune, Maharashtra, India

Prof. Karan Bahadur Shah, Budhanilakantha Municipality, Kathmandu, Nepal

Dr. Susan Cheyne, Borneo Nature Foundation International, Palangkaraja, Indonesia

Dr. Hemanta Kafley, Wildlife Sciences, Tarleton State University, Texas, USA

\section{Other Disciplines}

Dr. Aniruddha Belsare, Columbia MO 65203, USA (Veterinary)

Dr. Mandar S. Paingankar, University of Pune, Pune, Maharashtra, India (Molecular)

Dr. Jack Tordoff, Critical Ecosystem Partnership Fund, Arlington, USA (Communities)

Dr. Ulrike Streicher, University of Oregon, Eugene, USA (Veterinary)

Dr. Hari Balasubramanian, EcoAdvisors, Nova Scotia, Canada (Communities)

Dr. Rayanna Hellem Santos Bezerra, Universidade Federal de Sergipe, São Cristóvão, Brazil

Dr. Jamie R. Wood, Landcare Research, Canterbury, New Zealand

Dr. Wendy Collinson-Jonker, Endangered Wildlife Trust, Gauteng, South Africa

Dr. Rajeshkumar G. Jani, Anand Agricultural University, Anand, Gujarat, India

Dr. O.N. Tiwari, Senior Scientist, ICAR-Indian Agricultural Research Institute (IARI), New

Delhi, India

Dr. L.D. Singla, Guru Angad Dev Veterinary and Animal Sciences University, Ludhiana, India

Dr. Rupika S. Rajakaruna, University of Peradeniya, Peradeniya, Sri Lanka

Dr. Bahar Baviskar, Wild-CER, Nagpur, Maharashtra 440013, India

Reviewers 2018-2020

Due to pausity of space, the list of reviewers for 2018-2020 is available online.
The opinions expressed by the authors do not reflect the views of the Journal of Threatened Taxa, Wildlife Information Liaison Development Society, Zoo Outreach Organization, or any of the partners. The journal, the publisher, the host, and the partners are not responsible for the accuracy of the political boundaries shown in the maps by the authors.

\footnotetext{
Print copies of the Journal are available at cost. Write to:

The Managing Editor, JoTT,

c/o Wildlife Information Liaison Development Society,

No. 12, Thiruvannamalai Nagar, Saravanampatti - Kalapatti Road,

Saravanampatti, Coimbatore, Tamil Nadu 641035, India

ravi@threatenedtaxa.org
} 


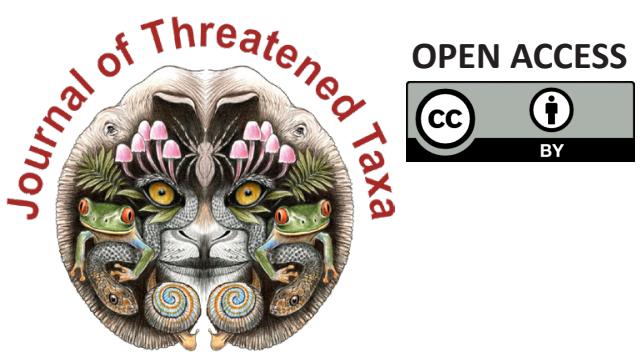

www.threatenedtaxa.org

The Journal of Threatened Taxa (JoTT) is dedicated to building evidence for conservation globally by publishing peer-reviewed articles online every month at a reasonably rapid rate at www.threatenedtaxa.org. All articles published in JoTT are registered under Creative Commons Attribution 4.0 International License unless otherwise mentioned. JoTT allows allows unrestricted use, reproduction, and distribution of articles in any medium by providing adequate credit to the author(s) and the source of publication.

\section{ISSN $0974-7907$ (Online) | ISSN $0974-7893$ (Print)}

\section{September 2021 | Vol. 13 | No. 11 | Pages: 19431-19674 Date of Publication: 26 September 2021 (Online \& Print) DOI: 10.11609/jott.2021.13.11.19431-19674}

Articles

Understanding human-flying fox interactions in the Agusan Marsh Wildlife Sanctuary as basis for conservation policy interventions

- Sherryl L. Paz \& Juan Carlos T. Gonzalez, Pp. 19431-19447

Argentinian odonates (dragonflies and damselflies): current and future distribution and discussion of their conservation

- A. Nava-Bolaños, D.E. Vrech, A.V. Peretti \& A. Córdoba-Aguilar, Pp. 19448-19465

\section{Communications}

The diel activity pattern of small carnivores of Western Ghats, India: a case study at Nelliampathies in Kerala, India

- Devika Sanghamithra \& P.O. Nameer, Pp. 19466-19474

Distribution and threats to Smooth-Coated Otters Lutrogale perspicillata (Mammalia: Carnivora: Mustelidae) in Shuklaphanta National Park, Nepal

- Gopi Krishna Joshi, Rajeev Joshi \& Bishow Poudel, Pp. 19475-19483

Wildlife hunting practices of the Santal and Oraon communities in Rajshahi, Bangladesh - Azizul Islam Barkat, Fahmida Tasnim Liza, Sumaiya Akter, Ashikur Rahman Shome \& M. Fazle Rabbe, Pp. 19484-19491

Ethnozoological use of primates in northeastern India

- Deborah Daolagupu, Nazimur Rahman Talukdar \& Parthankar Choudhury, Pp. 19492-19499

Factors influencing the flush response and flight initiation distance of three owl species in the Andaman Islands

- Shanmugavel Sureshmarimuthu, Santhanakrishnan Babu, Honnavalli Nagaraj Kumara \& Nagaraj Rajeshkumar, Pp. 19500-19508

Birds of Barandabhar Corridor Forest, Chitwan, Nepal

- Saneer Lamichhane, Babu Ram Lamichhane, Kapil Pokharel, Pramod Raj Regmi, Tulasi Prasad Dahal, Santosh Bhattarai, Chiranjibi Prasad Pokheral, Pabitra Gotame,

Trishna Rayamajhi, Ram Chandra Kandel \& Aashish Gurung, Pp. 19509-19526

On some additions to the amphibians of Gunung Inas Forest Reserve, Kedah,

Peninsular Malaysia

- Shahriza Shahrudin, Pp. 19527-19539

Reviews

A review of research on the distribution, ecology, behaviour, and conservation of the Slender Loris Loris lydekkerianus (Mammalia: Primates: Lorisidae) in India

- Mewa Singh, Mridula Singh, Honnavalli N. Kumara, Shanthala Kumar, Smitha D. Gnanaolivu \& Ramamoorthy Sasi, Pp. 19540-19552

Bivalves (Mollusca: Bivalvia) in Malaysian Borneo: status and threats

- Abdulla-Al-Asif, Hadi Hamli, Abu Hena Mustafa Kamal, Mohd Hanafi Idris, Geoffery James Gerusu, Johan Ismail \& Muyassar H. Abualreesh, Pp. 19553-19565

Disentangling earthworm taxonomic stumbling blocks using molecular markers

- Azhar Rashid Lone, Samrendra Singh Thakur, Nalini Tiwari, Olusola B. Sokefun \&

Shweta Yadav, Pp. 19566-19579

A reference of identification keys to plant-parasitic nematodes (Nematoda: Tylenchida) Tylenchomorpha)

- Reza Ghaderi, Manouchehr Hosseinvand \& Ali Eskandari, Pp. 19580-19602

Short Communications

Catalogue of herpetological specimens from Meghalaya, India at the Salim Ali Centre for Ornithology and Natural History

-S.R. Chandramouli, R.S. Naveen, S. Sureshmarimuthu, S. Babu, P.V. Karunakaran \&

Honnavalli N. Kumara, Pp. 19603-19610
A preliminary assessment of odonate diversity along the river Tirthan, Great Himalayan National Park Conservation Area, India with reference to the impact of climate change - Amar Paul Singh, Kritish De, Virendra Prasad Uniyal \& Sambandam Sathyakumar, Pp. 19611-19615

A checklist of orthopteran fauna (Insecta: Orthoptera) with some new records in the cold arid region of Ladakh, India

- M. Ali, M. Kamil Usmani, Hira Naz, Tajamul Hassan Baba \& Mohsin Ali, Pp. 19616-19625

New distribution records of two Begonias to the flora of Bhutan

- Phub Gyeltshen \& Sherab Jamtsho, Pp. 19626-19631

Rediscovery of Aponogeton lakhonensis A. Camus (Aponogetonaceae): a long-lost aquatic plant of India

- Debolina Dey, Shrirang Ramchandra Yadav \& Nilakshee Devi, Pp. 19632-19635

Glyphochloa acuminata (Hack.) Clayton var. laevis (Poaceae): a new variety from central Western Ghats of Karnataka, India

- H.U. Abhijit \& Y.L. Krishnamurthy, Pp. 19636-19639

A cytomorphological investigation of three species of the genus Sonchus L. (Asterales: Asteraceae) from Punjab, India

- M.C. Sidhu \& Rai Singh, Pp. 19640-19644

Dryopteris lunanensis (Dryopteridaceae) - an addition to the pteridophytic diversity of India

- Chhandam Chanda, Christopher Roy Fraser-Jenkins \& Vineet Kumar Rawat, Pp. 1964519648

Notes

First record of Spotted Linsang Prionodon pardicolor (Mammalia: Carnivora:

Prionodontidae) with photographic evidence in Meghalaya, India

- Papori Khatonier \& Adrian Wansaindor Lyngdoh, Pp. 19649-19651

First record of the Eastern Cat Snake Boiga gocool (Gray, 1835) (Squamata: Colubridae) from Tripura, India

- Sumit Nath, Biswajit Singh, Chiranjib Debnath \& Joydeb Majumder, Pp. 19652-19656

First record of the genus Tibetanja (Lepidoptera: Eupterotidae: Janinae) from India - Alka Vaidya \& H. Sankararaman, Pp. 19657-19659

Austroborus cordillerae (Mollusca: Gastropoda) from central Argentina: a rare, little-known land snail

- Sandra Gordillo, Pp. 19660-19662

Intestinal coccidiosis (Apicomplexa: Eimeriidae) in a Himalayan Griffon Vulture Gyps himalayensis

- Vimalraj Padayatchiar Govindan, Parag Madhukar Dhakate \& Ayush Uniyal, Pp. 1966319664

Two new additions to the orchid flora of Assam, India

- Sanswrang Basumatary, Sanjib Baruah \& Lal Ji Singh, Pp. 19665-19670

Wildlife art and illustration - combining black and white ink drawings with colour: some experiments in Auroville, India

- M. Eric Ramanujam \& Joss Brooks, Pp. 19671-19674
Publisher \& Host

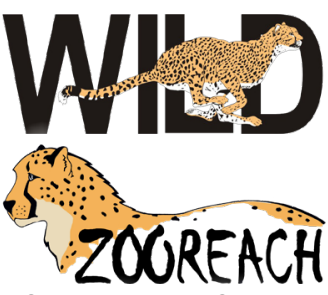

